\title{
Topographic Forcing from East Asia and North America in the Northern Winter Stratosphere and Their Mutual Interference
}

\author{
RONGCAI REN \\ State Key Laboratory of Numerical Modeling for Atmospheric Sciences and Geophysical Fluid Dynamics (LASG), \\ Institute of Atmospheric Physics, Chinese Academy of Sciences, Beijing, and Collaborative \\ Innovation Center on Forecast and Evaluation of Meteorological Disasters, Nanjing University \\ of Information Science and Technology, Nanjing, China
}

XIN XIA

State Key Laboratory of Numerical Modeling for Atmospheric Sciences and Geophysical Fluid Dynamics (LASG), Institute of Atmospheric Physics, Chinese Academy of Sciences, Beijing, China

JIAN RAO

Key Laboratory of Meteorological Disaster, Ministry of Education/Joint International Research Laboratory of Climate and Environment Change/Collaborative Innovation Center on Forecast and Evaluation of Meteorological Disasters, Nanjing University of Information Science and Technology, Nanjing, China

(Manuscript received 7 February 2019, in final form 9 September 2019)

\begin{abstract}
This study uses the stratosphere-resolved Whole Atmosphere Community Climate Model to demonstrate the "independent" and "dependent" topographic forcing from the topography of East Asia (EA) and North America (NA), and their "joint" forcing in the northern winter stratosphere. The mutual interference between the EA and NA forcing is also demonstrated. Specifically, without EA, an independent NA can also, like EA, induce a severe polar warming and weakening of the stratospheric polar vortex. While EA favors a displacement of the polar vortex toward Eurasia, NA favors a displacement toward the North AmericaAtlantic region. However, the independent-EA-forced weakening effect on the polar vortex can be largely decreased and changes to a location displacement when NA exists, and the interference the other way around is even more critical, being able to completely offset the independent-NA-forced effect, because EA can substantively obstruct NA's effect on the tropospheric wave pattern over the Eurasia-Pacific region. The much stronger (weaker) interference of EA (NA) is associated with its stronger (weaker) downstream weakening effect on the zonal flow that impinges on NA (EA). The mutual interference always tends to further destruct the upward wave fluxes over the eastern North Pacific and enhance the downward wave fluxes over North America. The overall changes in upward wave fluxes, as well as that in the Rossby stationary wavenumber responsible for the stratospheric changes, are related to changes in the zonal-mean flow pattern. The joint effects of EA and NA, rather than being a linear superimposition of their independent effects, are largely dominated by the effects of EA.
\end{abstract}

\section{Introduction}

In northern winters, the intensity as well as the spatial patterns of the stratospheric polar vortex is known to be closely related to the recurrent circulation patterns, such as the Arctic Oscillation or the North Atlantic Oscillation in the extratropical troposphere. The Arctic

Corresponding author: Rongcai Ren, rrc@lasg.iap.ac.cn
Oscillation and North Atlantic Oscillation are in turn good indicators of the weather and climate anomalies in the extratropics (Baldwin and Dunkerton 1999, 2001; Cai 2003; Lott et al. 2005; Chen et al. 2013; Yu et al. 2014; Cai et al. 2016). Increasing knowledge of the formation and variabilities of the stratospheric polar vortex has brought us new opportunities for fully understanding the climate patterns and their changes in recent decades. Apart from the meridional gradient of solar radiation 
between the low and high latitudes, planetary waves from the troposphere are another factor responsible for the distribution and the recurrent oscillations of the stratospheric polar vortex. Stationary planetary waves due to land-sea thermal contrast and topographic effects can largely determine the zonal asymmetries of the circulation pattern in the troposphere (Kasahara and Washington 1971; Lin 1982; Chen and Trenberth 1988; Valdes and Hoskins 1991; Ting et al. 2001; Held et al. 2002; Chang 2009), and are also the dominant components of the total planetary waves in the stratosphere (Smagorinsky 1953; Jacqmin and Lindzen 1985; Nigam et al. 1986, 1988).

By using simple models to perform idealized numerical experiments, early investigators identified the important dynamical roles of topographic forcing in modulating the stratosphere. For example, early simulations by Manabe and Terpstra (1974) indicated that topographic forcing serves to amplify the amplitudes of the stratospheric planetary waves of very low wavenumber, by transporting energy from the troposphere to the stratosphere. Later studies further demonstrated the varying dynamical roles of topography of different height (Taguchi and Yoden 2002; Gerber and Polvani 2009), location (Ringler and Cook 1995), and shape (Nakamura 1978; Luo et al. 1985; Ding 1992; Yanai and $\mathrm{Wu} 2006$ ) in modulating the planetary waves in the extratropical troposphere, and even the related stratospheric variabilities (Taguchi and Yoden 2002; Gerber and Polvani 2009). Specifically, the Northern Hemisphere-like variability with moderately frequent stratospheric sudden warmings can be observed in a simple model when the topographic height is in a certain range (Gerber and Polvani 2009). Topography located in midlatitudes has been found to be the dominant forcing of stationary planetary waves (Ringler and Cook 1995). Because of the generally zonal form of the Tibetan Plateau but the meridional form of the Rocky Mountains in the Northern Hemisphere, as well as their different altitudes, the westerly flows tend to be deflected horizontally when they meet the Tibetan Plateau, but vertically when they meet the Rocky Mountains, based on theoretical (Valdes and Hoskins 1991; Held et al. 2002; White et al. 2017) and observational (Luo et al. 1985; Ding 1992; Yanai and $\mathrm{Wu} 2006$ ) analyses. The different deflections of the zonal flow determine the contrasting topographic effects on the large-scale planetary-wave troughs and ridges between the Tibetan Plateau and the Rocky Mountains (Luo et al. 1985; Ding 1992; Yanai and Wu 2006). In addition, the different slopes of the topographic surfaces between the two regions may also matter for their deflection effects on the westerly flow
(Nakamura 1978; Luo et al. 1985; Valdes and Hoskins 1991; Cook and Held 1992; White et al. 2017), and thus for the distribution and amplitudes of the related stationary planetary waves. Compared with the troposphere, the stratosphere was found to be much more sensitive to topographic forcing (Jacqmin and Lindzen 1985).

Modeling studies indicate that a moderately strong stratospheric polar vortex with a realistic frequency of sudden stratospheric warmings can be reproduced with simple idealized surface topography of zonal wavenumber 1 (Taguchi and Yoden 2002) or wavenumber 2 (Gerber and Polvani 2009). Particularly, the model simulations carried out by Held (1983) suggest that the amplitudes of the planetary waves forced by the topography of the Eastern Hemisphere [or East Asia (EA)] are much stronger, and the pattern of the planetary waves resembles the observed one to a much larger extent than that forced by the topography of the Western Hemisphere [or North America (NA)]. This indicates the possibility of a crucial role played by the topography of EA in modulating the stratospheric circulation and its variabilities. Zou et al. (1991a,b) also proved the existence of a relatively stronger (weaker) upward propagation of planetary waves into the stratosphere in the Tibetan Plateau (Rocky Mountains) region, through simulations with a hemispheric multilevel model. They indicated that the differences can be attributed to the much higher latitudes to which the Rocky Mountains extend compared to the Tibetan Plateau. On the other hand, the much stronger topographic forcing from EA than that from NA may also be related to the joint forcing effects of the Tibetan Plateau and the Mongolian Plateau, connected to the north of the Tibetan Plateau, which have been shown to be significantly stronger in the extratropical troposphere than those of a single Tibetan Plateau or a single Mongolian Plateau (White et al. 2017; Shi et al. 2015).

However, due to the limited abilities of models in earlier simulations to reproduce the stratospheric variabilities, the particular topographic forcing from EA and from NA in modulating the stratospheric circulation pattern and its variabilities are still in need of further investigations through the use of more comprehensive, stratosphere-resolving models. Recently, White et al. (2018), using the Whole Atmosphere Community Climate Model (WACCM), showed the much stronger impacts of the Asian plateaus on the stratosphere than those of the American Rockies. Early linear and nonlinear model studies indicated that the atmospheric responses to topographic forcing are highly dependent on the low-level zonal flow pattern, and the topographic forcing in the region of Rockies can be influenced by the remote downstream effects of the Tibetan 
Plateau (Held and Ting 1990; Valdes and Hoskins 1991). On the other hand, the existence of the Rocky Mountains may help to extend the wavelength of planetary waves through its strengthening effect on the background zonal flow across the Northern Hemisphere (Luo et al. 1985), thus exhibiting a possible influence on the topographic forcing in other regions. With regard to the possible interactions of the topographic forcing between the plateaus of EA and the mountains of NA, in the current study we focus not only on the "joint" (combined mountains of NA and EA) and "dependent" (for the mountains of NA or EA when the other is present) topographic effects, but also the "independent" (for the mountains of NA or EA when the other is absent) topographic effects in the extratropical stratosphere. The terms "dependent" and "independent" here are respectively similar to the terms "separate" and "isolated" used by Held et al. (2002) when discussing the nonlinear responses to different topographies. By distinguishing the so-called dependent from independent effects of the mountains of NA and the plateaus of EA, the possible interactions between them can be further investigated, for which there is little in the literature but needed for a full understanding of the topographic effects in the extratropical stratosphere.

Recent progress in model reproducibility of stratospheric processes (Watanabe et al. 2008; Richter et al. 2010; Limpasuvan et al. 2012) has made further specific investigations on these issues far more practically possible. In this study, we carry out a series of numerical experiments with WACCM, forced under different topographic conditions, and perform deliberate parallel intercomparisons of the simulation results to demonstrate the joint, dependent, and independent (as defined above) topographic effects of the plateaus of EA and the Rocky Mountains of NA, as well as their possible interactions in their dynamical roles in modulating the stratosphere. The specific scientific questions we seek to address include the following: 1) What are the similarities and differences in the topographic effects from the plateaus of EA and the mountains of NA in the winter stratosphere? 2) What are the possible interactions between them in their topographic forcing in the stratosphere? 3) Which tends to dominate in their overall topographic effects in the winter stratosphere? 4) How can we understand the similarities or differences in their topographic effects and the possible interactions? Answers to these questions can benefit our current understanding of the patterns and variabilities in the stratosphere and help to extend our knowledge on the climate effects of the plateaus of EA and the Rocky Mountains of NA.

The rest of the paper is organized as follows. Section 2 introduces the model, the experimental design, and analysis methods; section 3 demonstrates the topographic effects and their mutual interference in modulating the circulation patterns in the stratosphere and troposphere. In section 4, we focus on the planetary-wave patterns and propagations forced by the topography to provide an understanding of the facts shown in section 3. A summary and further discussion are provided in section 5 .

\section{Model experiments and analysis methods}

\section{a. Model experiments}

We use version 4 of WACCM, the atmospheric component of NCAR's Community Earth System Model. It has 66 vertical levels, with a high model top up to $5.1 \times$ $10^{-6} \mathrm{hPa}$ (approximately $145 \mathrm{~km}$ ), and a horizontal resolution of $1.9^{\circ} \times 2.5^{\circ}$ (latitude $\times$ longitude). Compared with previous versions, the parameterization of topographic and nontopographic gravity waves has been improved (Garcia et al. 2007; Richter et al. 2010; Marsh et al. 2013), which has resulted in a much better performance in simulating the stratosphere and its variabilities (Garcia et al. 2007; Richter et al. 2010; de la Torre et al. 2012; Limpasuvan et al. 2012). As one of the best stratosphere-resolved models (Charlton-Perez et al. 2013; Rao et al. 2015), WACCM has been widely used to investigate various stratospheric problems, including long-term changes (Garcia et al. 2007), ozone hole recovery (Eyring et al. 2007, 2010), and atmospheric responses to solar forcing (e.g., Marsh et al. 2007; Jackman et al. 2009; Matthes et al. 2010) and tropical ENSO (e.g., Sassi et al. 2004; Taguchi and Hartmann 2006; Calvo et al. 2010; Garfinkel et al. 2010; Smith et al. 2011; Rao and Ren 2016a,b; Ren et al. 2017).

Since the concern in this study is the stationary atmospheric responses to topographic forcing in boreal winter, we run the model with a perpetual 15 January forcing to obtain a winter climatology more effectively. Also, we use a coupled land-atmosphere configuration, but the ocean is prescribed with the January climatology of the global sea surface temperature and sea ice derived from the HadISST dataset (Rayner et al. 2003). The original topographic data used in WACCM are from the United States Geological Survey dataset. In total, four experiments are carried out, including a control run (CTL) with realistic topographic forcing, and three sensitivity experiments in which either the plateaus of EA (noEA) or the Rocky Mountains of NA (noNA), or both (noEA\&noNA), are flattened to an altitude of $500 \mathrm{~m}$ (see Table 1). Figure 1 displays the topography maps of all the experiments. It can be seen that, by flattening the topography to $500 \mathrm{~m}$, we can guarantee a relatively smooth transition from the topography region to the surrounding regions, and meanwhile guarantee that the 
TABLE 1. List of the numerical experiments and their related settings.

\begin{tabular}{ll}
\hline \hline Name of experiment & \multicolumn{1}{c}{ Regions flattened } \\
\hline CTL & None \\
noEA & $50^{\circ}-120^{\circ} \mathrm{E}, 20^{\circ}-60^{\circ} \mathrm{N}$ \\
noNA & $90^{\circ}-150^{\circ} \mathrm{W}, 20^{\circ}-70^{\circ} \mathrm{N}$ \\
noEA\&noNA & $50^{\circ}-120^{\circ} \mathrm{E}, 20^{\circ}-60^{\circ} \mathrm{N}$ and $90^{\circ}-150^{\circ} \mathrm{W}$, \\
& $20^{\circ}-70^{\circ} \mathrm{N}$ \\
\hline
\end{tabular}

flattened regions are generally the main part of the plateaus. For consistency of altitude in the flattened regions, we always flatten to $500 \mathrm{~m}$ in both EA and NA, although the Tibetan Plateau in EA (over $4500 \mathrm{~m}$ ) was originally much higher than the Rocky Mountains of NA (over 2000-3000 m). This choice might lead to slightly weaker impacts of NA's topography than from lower flattening, but it will effectively guarantee that the flow patterns are forced by the main body of the topography. The SGH and SGH30 parameters needed in WACCM for nonresolved gravity waves are set to be 150 and $50 \mathrm{~m}$ for the flattened regions to represent the reduced gravity wave effects. All four experiments are integrated for 6570 days with a perpetual January forcing, and the last 5475 days are used to derive the winter climatology for further analyses. For brevity, below, we use "EA" and "NA" to denote the plateaus of EA and the mountains of NA, respectively, in most circumstances.

\section{b. Analysis method}

\section{1) DEPENDENT, INDEPENDENT, AND JOINT TOPOGRAPHIC EFFECTS}

Based on the arguments in the introduction, we define the dependent topographic effects of (or the stationary atmospheric responses to) EA/NA as the differences in the model-produced winter climatology between experiment CTL and noEA/noNA (effects of EA when NA exists, or effects of NA when EA exists); the independent topographic effects of EA as the differences between experiment noNA and noEA\&noNA (effects of EA without NA); the independent topographic effects of NA as the differences between experiment noEA and noEA\&noNA (effects of NA without EA); and their joint topographic effects as the differences between CTL and noEA\&noNA, respectively. Furthermore, by comparing the dependent with the independent effects defined above, we identify the possible topographic interactions between EA and NA. To facilitate understanding of the results from the above comparisons, we show in Fig. 2 a schematic layout of the following figures (except Fig. 6), including brief explanations of the experimental settings or the objectives of the corresponding panels.

\section{2) Diagnosis Methods}

To diagnose the upward wave activity responses to topographic forcing, we adopt the three-dimensional wave activity flux from Plumb (1985) to obtain the spatial distribution of the vertical component of the wave flux. It can be expressed as follows:

$$
\mathbf{F}_{z}=\frac{p \cos \phi}{1000 \mathrm{hPa}}\left[\frac{2 \Omega^{2} \sin ^{2} \phi}{N^{2} a \cos \phi}\left(\frac{\partial \psi^{\prime}}{\partial \lambda} \frac{\partial \psi^{\prime}}{\partial z}-\psi^{\prime} \frac{\partial^{2} \psi^{\prime}}{\partial \lambda \partial z}\right)\right],
$$

where $a$ is the radius of Earth; $\phi$ and $\lambda$ represent the latitude and longitude, respectively; $p$ is pressure; $z$ is the vertical coordinate in terms of log-pressure; $N$ denotes (a) noEA\&noNA

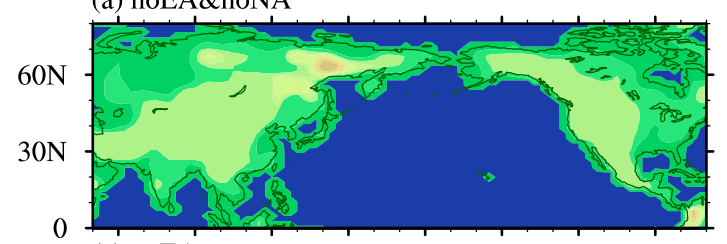

(c) noEA



(b) noNA

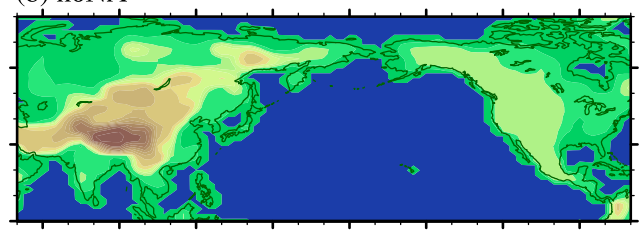

\section{(d) CTL}

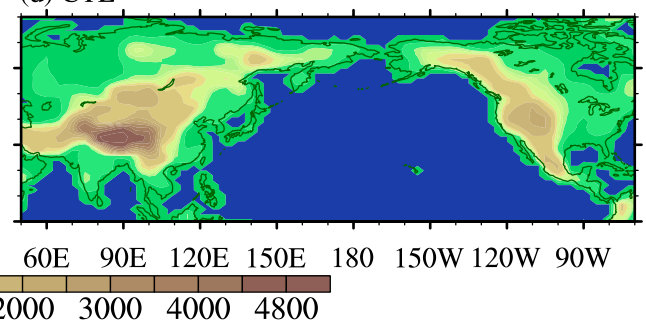

FIG. 1. Topography maps for the four numerical experiments: (a) noEA\&noNA, (b) noNA, (c) noEA, and (d) CTL. 


\begin{tabular}{|l|l|l|}
\hline $\begin{array}{l}\text { (a) noEA\&noNA } \\
\text { Both EA and NA } \\
\text { topography flattened }\end{array}$ & $\begin{array}{l}\text { (b) noNA } \\
\text { EA unchanged; } \\
\text { NA flattened. }\end{array}$ & $\begin{array}{l}\text { (c) } \text { Independent effect of } \\
\text { EA. }\end{array}$ \\
\hline \hline $\begin{array}{l}\text { (d) noEA } \\
\text { EA flattened; } \\
\text { NA unchanged. }\end{array}$ & $\begin{array}{l}\text { (e) CTL } \\
\text { Realistic topography }\end{array}$ & $\begin{array}{l}\text { (f) [(e) minus (d)] } \\
\text { Dependent effect of EA } \\
\text { when NA exists. }\end{array}$ \\
\hline \hline $\begin{array}{l}\text { (g) [(d) minus (a)] } \\
\text { Independent effect of } \\
\text { NA. }\end{array}$ & $\begin{array}{l}\text { (h) [(e) minus (b)] } \\
\text { Dependent effect of NA } \\
\text { when EA exists. }\end{array}$ & $\begin{array}{l}\text { (i) [(e) minus (a)] } \\
\text { Joint effect of EA\&NA }\end{array}$ \\
\hline
\end{tabular}

FIG. 2. Schematic layout of the following figures with brief explanations of the experimental settings shown in (a), (b), (d), and (e), and the objectives of (c), (f), (g), (h), and (i) in the following figures.

the buoyancy frequency; and $\psi^{\prime}$ represents the perturbed quasigeostrophic streamfunction relative to the zonal mean $[\psi]$.

The zonal-mean wave flux and its divergence are calculated based on Eliassen-Palm (EP) flux theory (Andrews and McIntyre 1976). The formulations are as follows:

$$
\begin{gathered}
\mathbf{F}=\left(\mathbf{F}_{y}, \mathbf{F}_{z}\right)=\left(-\rho_{0} a \cos \phi \overline{u^{\prime} v^{\prime}}, \rho_{0} a \cos \phi \frac{R f}{H N^{2}} \overline{v^{\prime} T^{\prime}}\right), \\
\mathbf{D}_{F}=\frac{\nabla \cdot \mathbf{F}}{\rho_{0} a \cos \phi}=\frac{\partial\left(\mathbf{F}_{y} \cos \phi\right) / a \cos \phi \partial \phi+\partial \mathbf{F}_{z} / \partial z}{\rho_{0} a \cos \phi}
\end{gathered}
$$

where $\rho_{0}$ is the air density, $R$ is the gas constant, $H$ is the mean scale height $(\sim 8 \mathrm{~km})$, and $f$ is the Coriolis parameter. Other parameters are the same as in Eq. (1).

To understand the propagation pathways of the stationary waves under different topographic conditions, we also diagnose the Rossby stationary wavenumber $k_{s}$ derived in the Mercator coordinate system (Hoskins and Karoly 1981; Hoskins and Ambrizzi 1993). The term $k_{s}$ can be used to display the distribution of the threshold wavenumber and thus infer the waveguides for stationary Rossby waves. It is calculated as follows:

$$
k_{s}=\left(\frac{\beta_{*}}{\bar{U}}\right)^{1 / 2}
$$

where $\beta_{*}$ is the meridional gradient of absolute vorticity and $\bar{U}$ is the zonal-mean zonal wind. The wave fluxes and the $k_{s}$ for all experiments are calculated from the climatological mean circulation fields, and their differences are used to indicate the topographic effects on the wavenumber.

\section{Topographic effects on the intensity and horizontal structure of the stratospheric polar vortex}

\section{a. On the zonal-mean intensity}

Figure 3 shows the zonal-mean temperature and zonal wind patterns in the four experiments (Figs. 3a,b,d,e) and their differences (Figs. 3c,f-i) between different experiments, which are displayed in a similar layout as in Fig. 2. First, we can confirm that the modelreproduced zonal-mean patterns largely resemble those reproduced by the reanalysis datasets [top panel of Fig. 1 in Rao et al. (2015)], as exhibited by the reasonable strength of the polar jet in the stratosphere and the subtropical jet in the upper troposphere, as well as the reasonably cold polar stratosphere and cold tropical tropopause (Figs. 3a,b,d,e). The differences between different experiments can be observed only from the slight changes of the intensity or the meridional location of the polar jet.

The zonal-mean pattern difference between experiment noNA and noEA\&noNA shows that, when NA is nonexistent, the independent effect of EA is to yield a severe weakening of the stratospheric polar jet (about $-13 \mathrm{~m} \mathrm{~s}^{-1}$ at $10 \mathrm{hPa}$ ), accompanied by a severe polar warming (over $7.0 \mathrm{~K}$ ) in the midstratospheric layer (Fig. 3c). The zonal-mean pattern difference between experiment CTL and noEA, however, shows that, when NA exists there, EA's dependent dynamical effects become much more trivial, exhibiting only a slight weakening (from -1 to $-2 \mathrm{~ms}^{-1}$ at $10 \mathrm{hPa}$ ) and equatorward-shifting effect on the stratospheric polar 
(a) noEA\&noNA

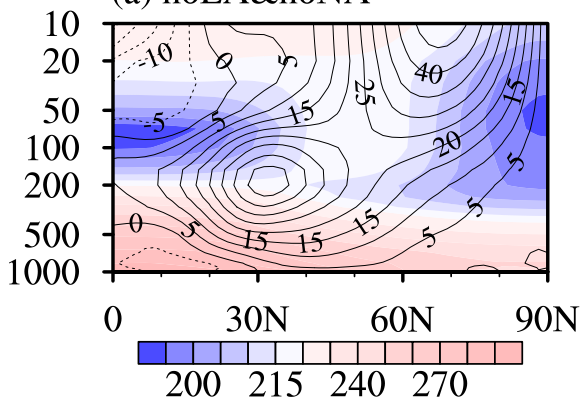

(d) noEA

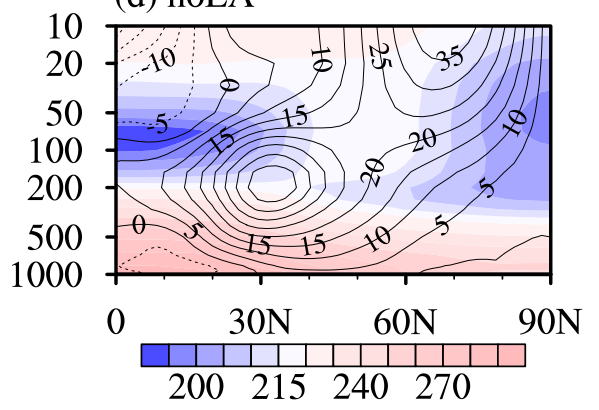

(g) (d)-(a)

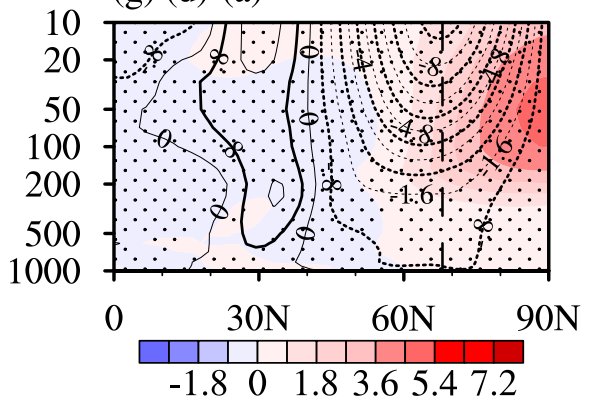

(b) noNA

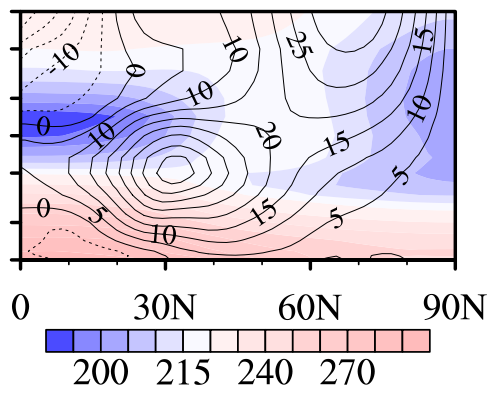

(e) CTL

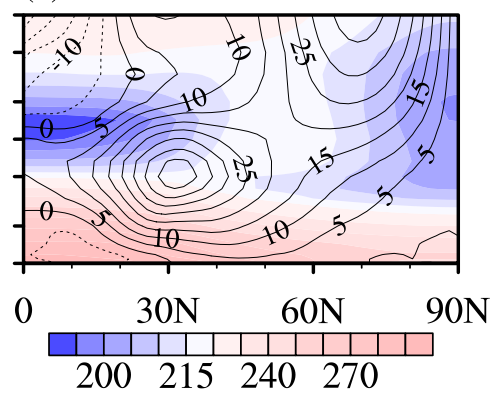

(h) (e)-(b)

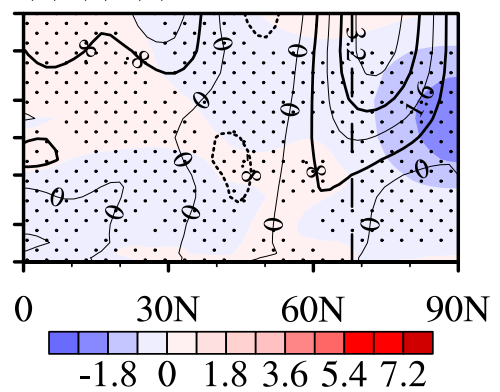

(c) (b)-(a)

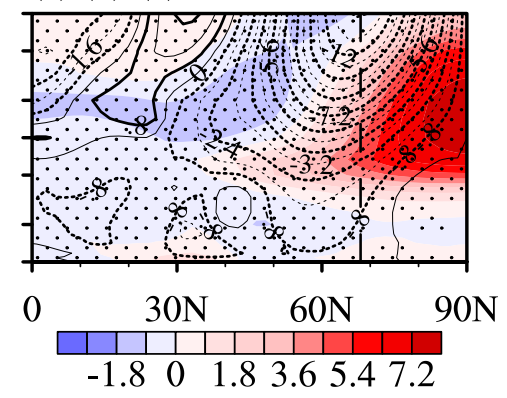

(f) (e)-(d)

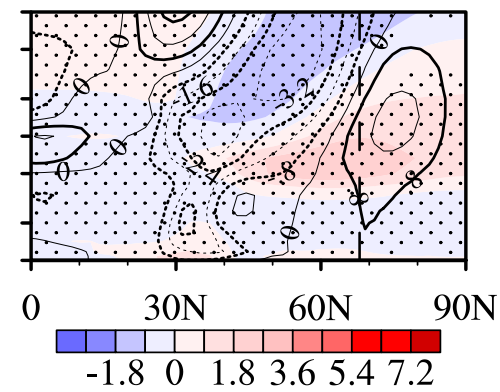

(i) (e)-(a)

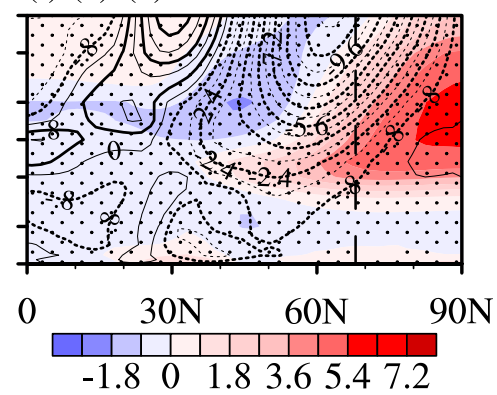

FIG. 3. The winter climatology of zonal-mean temperature (K; shading) and zonal wind [ $\mathrm{m} \mathrm{s}^{-1}$; contours; interval is 5 in (a), (b), (d), and (e) and 0.8 in other panels] in the Northern Hemisphere in (a) noEA\&noNA, (b) noNA, (d) noEA, and (e) CTL, and the differences (c) between noNA and noEA\&noNA, (f) between CTL and noEA, (g) between noEA and noEA\&noNA, (h) between CTL and noNA, and (i) between CTL and noEA\&noNA. Dotted areas in (c), (f), and (g)-(i) mark the 95\% confidence level from a Student's $t$ test of the differences in zonal-mean temperature. The vertical dashes mark the average location of the stratospheric polar jet based on the zonalmean zonal wind at $10 \mathrm{hPa}$. The color bars for (a), (b), (d), and (e) are different from those for other panels.

jet in the upper layer (above $50 \mathrm{hPa}$ ), but a slight strengthening $\left(1-2 \mathrm{~m} \mathrm{~s}^{-1}\right.$ at $\left.\sim 100 \mathrm{hPa}\right)$ and polewardshifting effect on the stratospheric polar jet in the lower layer (below $50 \mathrm{hPa}$ ) (Fig. 3f). This is accompanied by a weak polar cooling (within $-1 \mathrm{~K}$ ) in the upper stratosphere but a polar warming (within $+2 \mathrm{~K}$ ) in the lower stratosphere (Fig. 3f). The much weakened dynamical effects of EA due to the existence of NA clearly indicate a significant destructive interference by NA with EA's influences on the extratropical stratospheric circulation.

On the other hand, the zonal-mean pattern difference between experiment noEA and noEA\&noNA shown in Fig. 3g indicates that, similar to EA, NA's independent topographic effects also yield a severe weakening of the stratospheric polar jet (over $-8 \mathrm{~m} \mathrm{~s}^{-1}$ at $10 \mathrm{hPa}$ ), accompanied by a significant polar warming (over $4.0 \mathrm{~K}$ ) in the midstratospheric layer. When EA is not flattened, however, the dependent dynamical effects of NA, as represented by the difference between experiment CTL and noNA, show a reversed strengthening of the stratospheric polar jet $\left(\sim 3 \mathrm{~m} \mathrm{~s}^{-1}\right.$ at $\left.10 \mathrm{hPa}\right)$ accompanied by a cooling (approximately $-2 \mathrm{~K}$ ) in the midstratospheric polar region (Fig. 3h). This clearly indicates that EA's interference with NA's topographic effects is so strong and critical that it can completely offset the independent dynamical effects of NA. This very strong interference of EA with the impacts of NA presented here can indirectly explain why NA's topographic effects in the stratosphere or on the upward 
planetary-wave activity have usually been found to be much weaker and less significant compared with those of EA (Held 1983; Zou et al. 1991a,b; White et al. 2018). This is because the topographic effects investigated in previous studies were actually dependent ones. The current results suggest that, originally, NA's topographic effect in the stratosphere was never much weaker than, but comparable to, EA's if the interference does not occur. The offsetting interference from EA on NA's effects may also suggest a relatively dominant role played by EA in modulating the stratosphere.

The joint effects of EA and NA represented by the pattern difference between experiment CTL and noEA\&noNA are displayed in Fig. 3i. It can be seen that the overall joint weakening effects (over $-11 \mathrm{~m} \mathrm{~s}^{-1}$ at $10 \mathrm{hPa}$ ) on the stratospheric polar jet exhibit a highly similar zonal-mean pattern to EA's (but not NA's) independent effects, and are even slightly weaker than EA's independent effects (about $-13 \mathrm{~m} \mathrm{~s}^{-1}$ at $10 \mathrm{hPa}$ ) (Fig. 3c vs Fig. 3i; Fig. 3g vs Fig. 3i). This indicates there is indeed a dominance of EA over NA in their joint topographic effects on both the intensity and spatial pattern responses of the stratospheric polar vortex. In addition, rather than being a linear superimposition of their independent effects, the joint effects exhibit clear nonlinearity, probably due to the mutual interference between them. Another notable feature is that the EAforced zonal-mean pattern seems to exhibit a more "baroclinic" character, while the NA-forced one seems to have a more "barotropic" vertical structure in the meridional direction, as exhibited by the greater or lesser northward tilting of the axis of the anomalous zonal wind. We investigate this further in the next section.

In general, contrary to the known much weaker topographic effects of NA, it is found that the independent topographic effects of NA are comparably strong with those of EA in yielding a severe weakening of the stratospheric polar vortex. Their effects interfere with each other significantly, with the interfering effects of EA on NA being especially stronger than the other way around, explaining the relatively much stronger dependent effects of EA than NA. Furthermore, their joint topographic effects in the extratropical stratosphere exhibit clear nonlinearity and are largely dominated by EA's effects.

\section{b. On the horizontal structure of the stratospheric polar vortex}

To show the spatial pattern of the stratospheric polar vortex and its changes due to topographic forcing, we display in Fig. 4 the geopotential height and its zonal deviation at $10 \mathrm{hPa}$ in a similar layout as in Fig. 3.
First, a generally similar pattern of the polar vortex can be observed in the four experiments, as exhibited by the similar distributions of the zonal deviations of the geopotential height among them (Figs. 4a,b,d,e). In relation to the severe weakening of the polar vortex shown in Fig. 3c, the stratospheric polar vortex is mainly weakened (up to $+720 \mathrm{gpm}$ ) and, meanwhile, slightly shifted toward Eurasia, as seen from the dominant positive geopotential height changes over the polar region (Fig. 4c). When NA exists, however, EA's effects change to one of mainly shifting the polar vortex toward Eurasia and away from NA, as seen from the typical wavenumber-1-like pattern of the changes in both geopotential height and its zonal deviation (Fig. 4f). This seems to suggest that NA's existence interferes with EA's effects by mainly destructing the intensity weakening but largely increasing the location displacement of the polar vortex forced by EA.

On the other hand, NA's independent effects also mainly weaken the polar vortex (up to $+560 \mathrm{gpm}$ ) but meanwhile help to shift it toward the NA-Atlantic region and away from Eurasia (Fig. 4g), that is, in the opposite direction to that for EA's independent effects shown in Fig. 4c. It can be seen from Fig. 4h that the existence of EA also tends to dramatically interfere with NA's effects, and can change them to a shrinking of the polar vortex, as exhibited by the weaker negative geopotential height changes confined to the Atlanticnorthern Europe region (up to $-240 \mathrm{gpm}$ ) and the similar zonal deviation pattern to the climatological one in Figs. $4 \mathrm{~b}$ and $4 \mathrm{e}$. This confirms that the existence of EA can generally offset NA's weakening effect on the stratospheric polar vortex. It can be seen from Fig. $4 \mathrm{i}$ that the joint effects of EA and NA also exhibit a weakening of the polar vortex and a slight location displacement of it toward Eurasia, which is more (less) similar to EA's (NA's) independent effects shown in Figs. $4 \mathrm{c}$ and $4 \mathrm{~g}$. This again indicates the relative dominance of EA in the joint topographic forcing from the two regions. In general, both EA and NA act primarily to weaken and slightly shift the polar vortex, but the effects tend to be reduced when the other exists, especially for NA's effects, which can be offset due to the existence of EA.

\section{On the planetary-wave patterns and propagations in the extratropics}

\section{a. Wave patterns in the upper troposphere}

To understand the mutual interference between EA and NA in their topographic effects in the stratosphere, we next diagnose their forced changes in the flow 
(a) noEA\&noNA
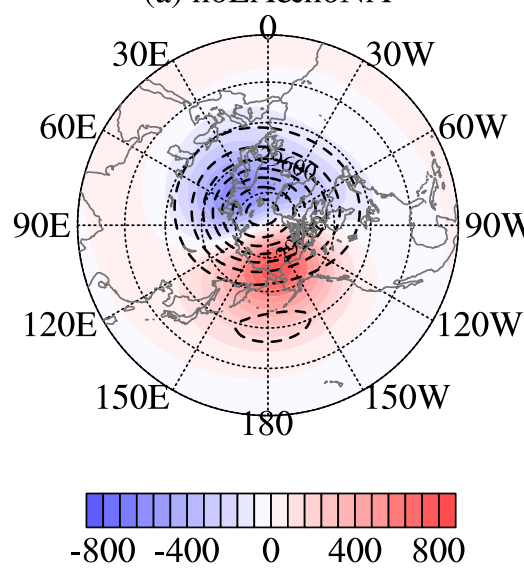

(d) noEA


(g) (d)-(a)
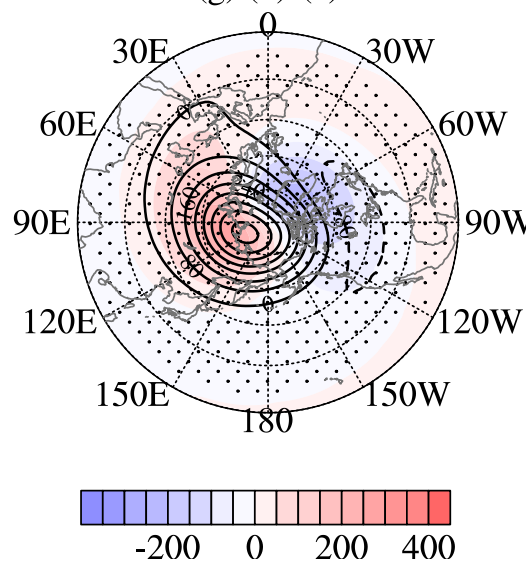

(b) noNA
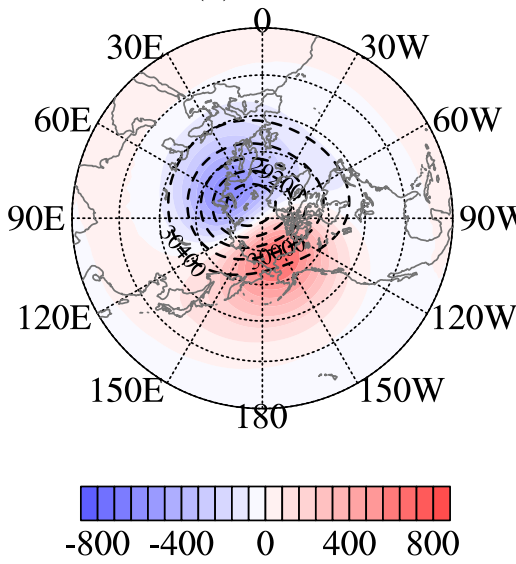

(e) CTL
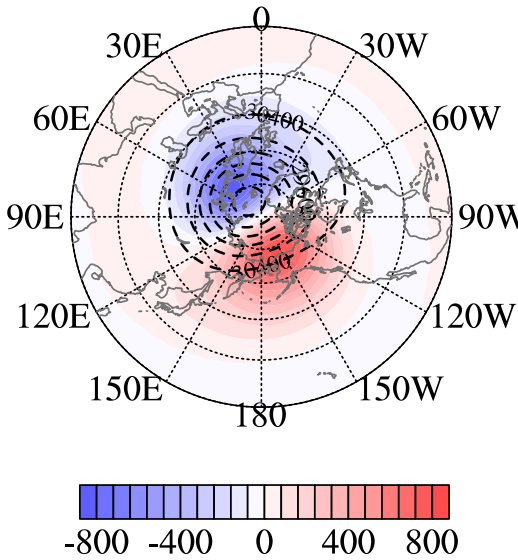

(h) (e)-(b)


(c) (b)-(a)
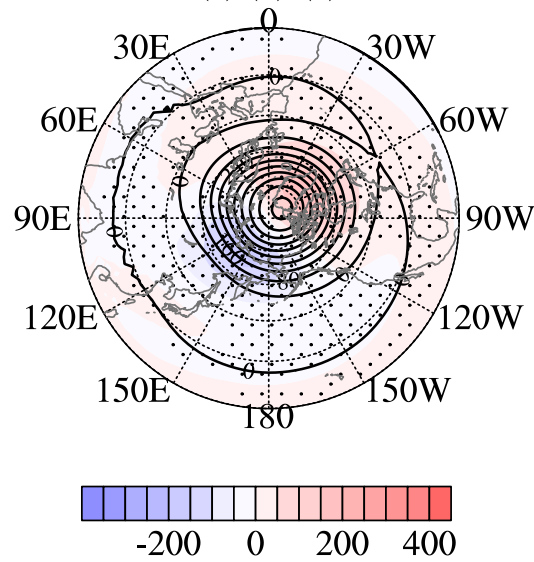

(f) (e)-(d)
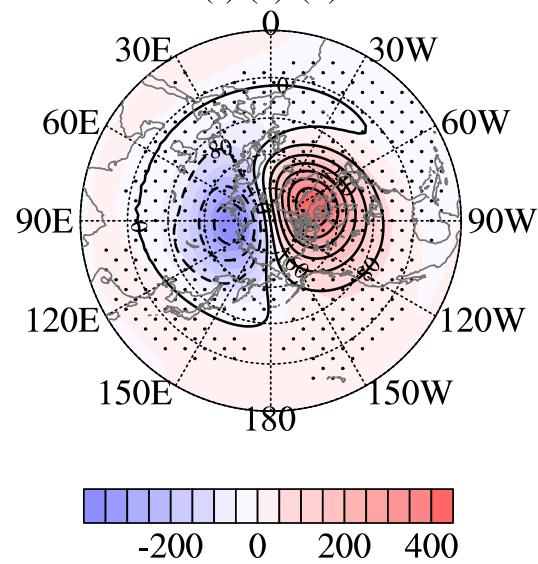

(i) (e)-(a)
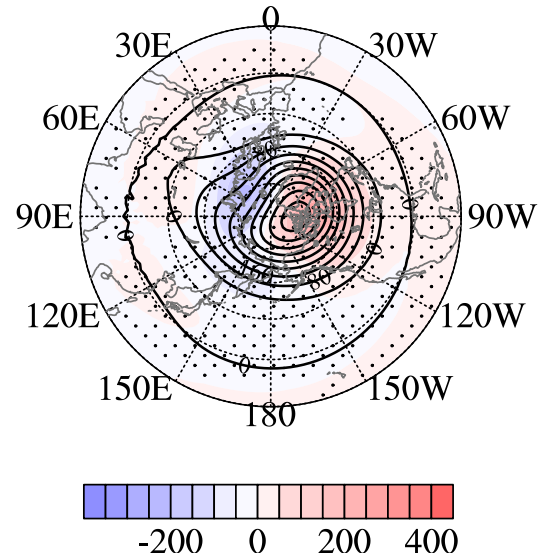

FIG. 4. As in Fig. 3, but for the 10-hPa geopotential height (gpm; contours) and its zonal deviations (shading) north of $15^{\circ} \mathrm{N}$. The contour interval in (a), (b), (d), and (e) is 400 and that in (c), (f), and (g)-(i) is 80 . Dotted areas in (c), (f), and (g)-(i) mark the 95\% confidence level from the Student's $t$ test of the differences in the geopotential height differences. The color bars for (a), (b), (d), and (e) are different from those for other panels.

pattern in the upper troposphere. Figure 5 shows the streamflow, the zonal deviation of geopotential height at $200 \mathrm{hPa}$, and their differences between different experiments. First, it is notable that all experiments consistently show a general wavenumber-2-like flow pattern in the extratropics, including the East Asian trough (EAT) extending from the Asian continent to the western Pacific, the North American ridge (NAR) 

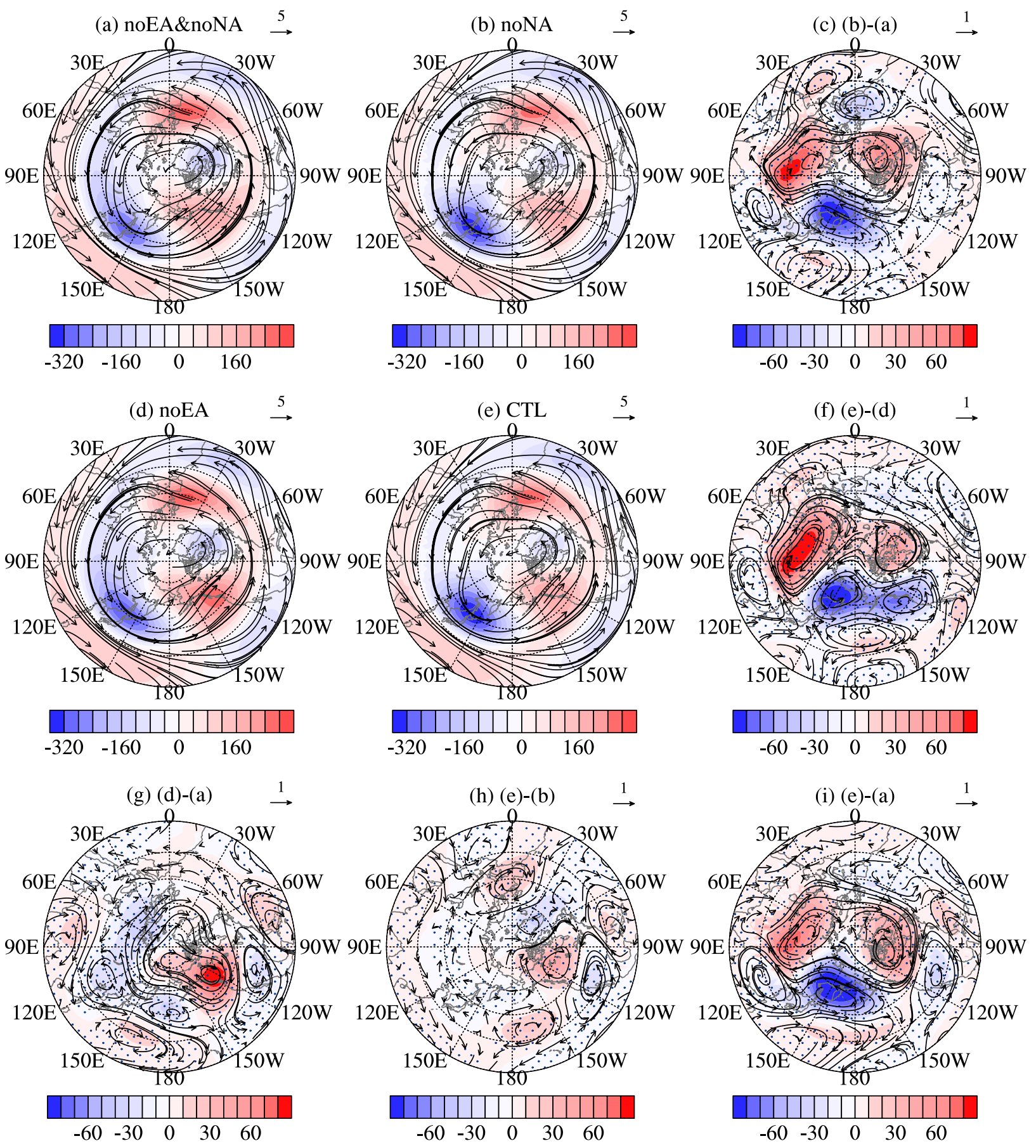

FIG. 5. As in Fig. 4, but for the 200-hPa wind flow ( $\mathrm{m} \mathrm{s}^{-1}$; stream vectors) and zonal deviation of geopotential height (gpm; shading). Dotted areas in (c), (f), and (g)-(i) mark the 95\% confidence level from the Student's $t$ test of the geopotential height differences. The color bars for (a), (b), (d), and (e) are different from those for other panels.

across the Rocky Mountains region from the east Pacific to continental NA, and the relatively narrower North American trough (NAT) over the eastern sector of the North American continent and the Atlantic height ridge (ATR) mainly over the Atlantic region (Figs. 5a,b,d,e).
It can be seen from Fig. $5 \mathrm{c}$ that EA's independent effect is to force an anticyclonic high anomaly center over the extratropical Eurasian continent, in relation to the horizontal deflection effect on the zonal flow to the north of EA's topography. The forced anticyclonic flow 
results in a weakened EAT over the Eurasian continent but a deepened EAT downstream over the western Pacific (or an eastward-shifted EAT), due to the anomalous northerlies in the east of the anticyclonic high center. This is also conducive to the EAT extending eastward to the northeastern Pacific, as manifested by the cyclonic low anomaly center over the entire North Pacific. This cyclonic anomaly center extends to the northwestern part of the North American continent and weakens the NAR, and thus the NAT (Fig. 5a vs Fig. 5c). Between the two anticyclonic high anomaly centers over the North American and Eurasian continents, there exists a cyclonic low anomaly center, which results in a weakened ATR (Fig. 5c). Overall, EA's independent effect favors a deepened and eastward-shifted EAT, accompanied by a weakened and eastward-shifted NAR, a weakened NAT, and a weakened ATR. We know that this is related to a severe weakening of the stratospheric polar vortex (Fig. 3c), which we discuss further in the following part.

When NA exists, the EA-forced anomalous flow pattern in the troposphere seems to exhibit a general wavenumber-1-like pattern, consisting of an even stronger and further zonally elongated anticyclonic high anomaly center across the Eurasian continent, two connected cyclonic low anomaly centers over the western North Pacific and the region from the eastern North Pacific to western NA, respectively, and an anticyclonic high anomaly center farther downstream that gets directly connected to the Eurasian high anomaly center (Fig. 5f). This results in the more apparent wavenumber-1-like pattern compared with the independent-EA-forced one in Fig. 5c. As a result, the EAT extends farther eastward, which helps to result in an additionally weakened NAR and a stillweakened NAT, but a less weakened ATR than that in Fig. 5c (Fig. 5f). This suggests that EA's effects on the tropospheric flow pattern may not be weakened very much due to the existence of NA, but the wavelength of the forced anomaly pattern seems to be slightly extended. This is consistent with the arguments in Luo et al. (1985) that the existence of NA can help to extend the circulation wavelength because of its strengthening effect on the background zonal flow. It is just the general wavenumber-1-like anomaly pattern from the troposphere to stratosphere that is related to the main location displacement of the stratospheric polar vortex in Fig. 4f.

It can be seen from Fig. $5 \mathrm{~g}$ that, when the forcing of NA is independent, its deflection effect induces an anticyclonic high anomaly center over the northwestern part of the North American continent, which enhances the NAR and spans over a large area by extending northeastward and northwestward. The northeastward extension of the NAR results in a less deepened NAT and a slightly enhanced ATR. Meanwhile, the southerlies to the west of the forced anticyclonic high anomaly center favor more warm air to be advected to the highlatitude North Pacific and even to the Arctic region, which corresponds to the anomalous easterlies in the high latitudes of the North Pacific (Xia et al. 2019). The development of these easterly anomalies thus helps the forced anticyclonic high anomaly center to extend farther northward and westward. This in turn favors cyclonic anomalies across the Eurasian continent and the western-to-central North Pacific, or a slightly deepened and eastward-extended EAT. Although the cyclonic anomalies over Eurasia and the western Pacific are relatively weaker than the anticyclonic anomalies over NA, the anomaly pattern in the zonal direction is wavenumber-1-like (Fig. 5g). We know from Figs. 3g and $4 \mathrm{~g}$ that this wave pattern corresponds to a significant weakening of the stratospheric polar vortex and polar jet. When EA exists, the effects of NA on the tropospheric circulation become largely weakened, and those over Eurasia are substantively blocked, as exhibited by the less modulated flow pattern in most regions of Eurasia and the North Pacific (Fig. 5h). NA's weaker effects are thus generally confined to a limited downstream region from NA to the Atlantic. This corresponds to the complete offset of NA's effects in the stratosphere due to the existence of EA.

Similar to the stratospheric circulation, the joint effects of EA and NA on the planetary wave pattern in the upper troposphere are also dominated by EA forcing, except for a less weakened ATR than under the independent-EA-forced condition (Fig. 5i vs Fig. 5c and Fig. 5i vs Fig. 5g). From Fig. 5h it can be seen that NA's effects do indeed favor a slight strengthening of the ATR.

To understand the more critical interference of EA with NA's effects on the wave pattern, we show in Fig. 6 the 500-hPa zonal wind changes due to different topographic forcing. It can be seen from Fig. $6 a$ that, besides a severe weakening of the zonal westerly flow (as seen from the forced strong easterly anomalies) in the subtropics and a strengthening of that (as seen from the westerly anomalies) in higher and lower latitudes over Eurasia due to the blocking and deflection effect of EA, the downstream effect of an independent EA can also yield a significant weakening of the zonal flow in higher (north of $50^{\circ} \mathrm{N}$ ) and lower latitudes (south of $15^{\circ} \mathrm{N}$ ) latitudes, and a strengthening of that in midlatitudes $\left(15^{\circ}-50^{\circ} \mathrm{N}\right)$ of the western-to-central North Pacific. We know from Fig. 5c that this downstream effect on the zonal flow is related to a deepened and 
(a)EA forced, without NA
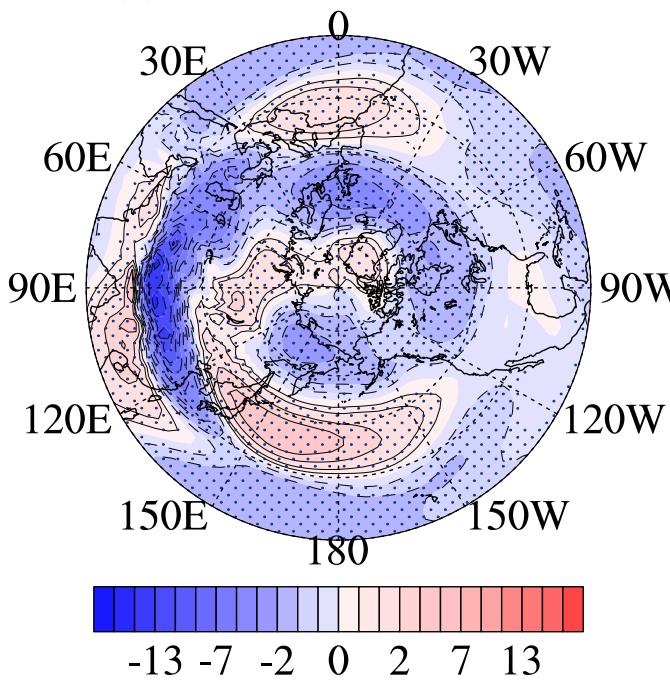

(c)NA forced, without EA
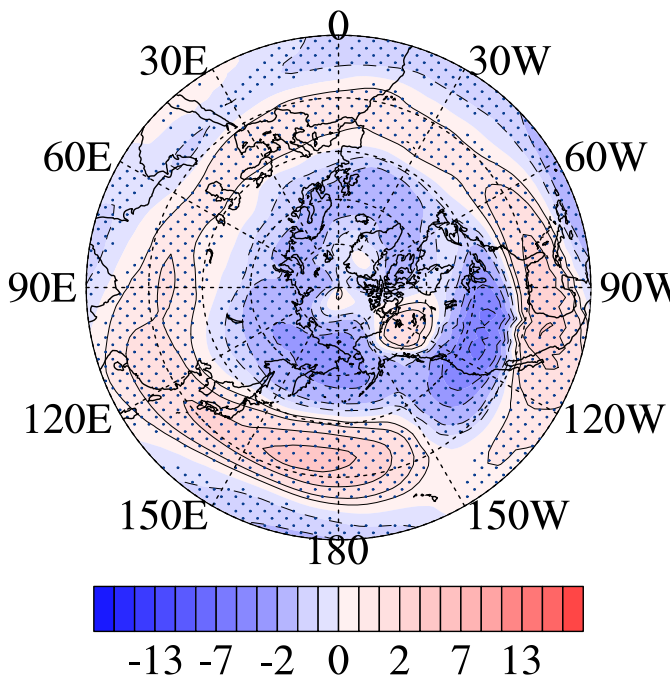

(b)NA forced, with EA

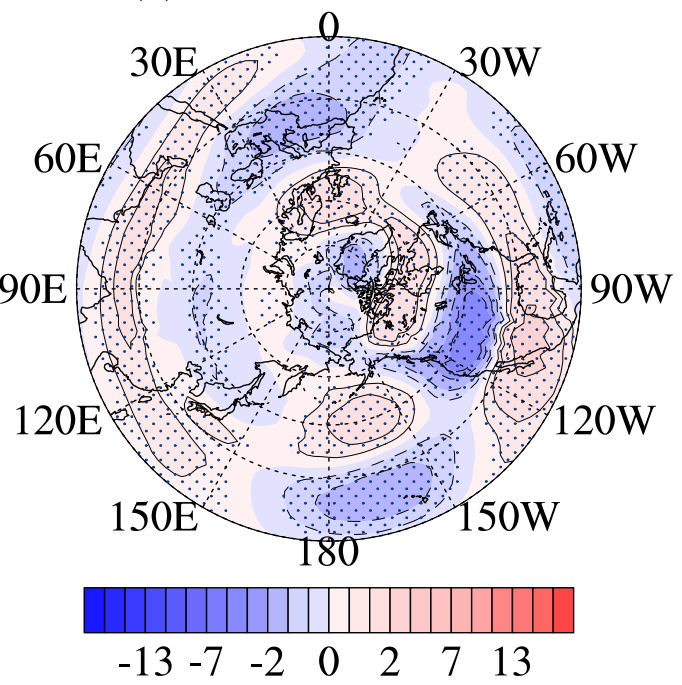

(d)EA forced, with NA

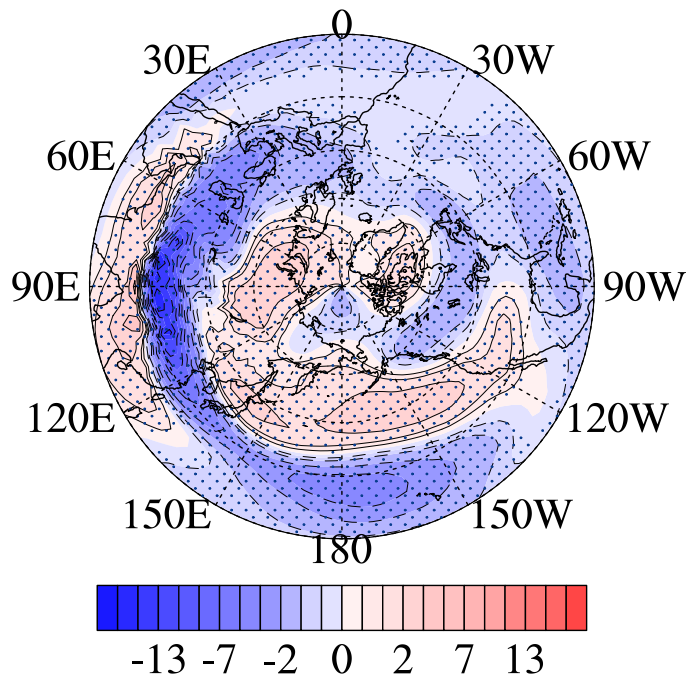

FIG. 6. The 500-hPa zonal wind changes $\left(\mathrm{m} \mathrm{s}^{-1}\right)$ in the Northern Hemisphere north of $15^{\circ} \mathrm{N}$, due to (a) the independent topographic forcing of EA, (b) the dependent topographic forcing of NA when EA exists, (c) the independent topographic forcing of NA, and (d) the dependent topographic forcing of EA when NA exists. The dotted areas indicate the $95 \%$ confidence level of the changes from the Student's $t$ test. The shading and contours have the same interval but the zero contour is omitted for clarity.

eastward-extended EAT over the North Pacific. On the downstream side of the eastward-extended EAT, southeasterly anomalies prevail upstream of the NA region (Fig. 5c), and significant easterly anomalies dominate upstream of the NA region (Fig. 6a). This implies that the background zonal flow that impinges on NA can be generally slowed down due to the existence of EA. As indicated in earlier studies (e.g., Held and Ting 1990; Ringler and Cook 1995; Held et al. 2002), the much-slowed-down zonal flow should help to reduce the topographic effect of NA. We observe from
Fig. 6 that, when EA exists, the blocking and deflection effect of NA does indeed become much weaker than that without EA (Fig. 6b vs Fig. 6c), especially for NA's downstream effect over Eurasia, as also seen from Fig. $5 \mathrm{~h}$. In contrast, due to the relatively much weaker topographic forcing of NA than EA, the local as well as downstream effect of an independent NA on the zonal flow over Eurasia is much weaker than that of an independent EA (Fig. 6a vs Fig. 6c). Furthermore, the weaker downstream effect of NA over Eurasia acts to weaken the zonal flow north of $50^{\circ} \mathrm{N}$, but it slightly 
strengthens the subtropical zonal flow that just impinges on EA (Fig. 6c). This implies that, when NA exists, EA's topographic effect will be partially weakened due to the slowed-down zonal flow north of $50^{\circ} \mathrm{N}$, and partially enhanced due to the strengthened zonal flow in the subtropics. This corresponds to the less interfered effect of EA by NA. Therefore, it is the relatively much stronger (weaker) downstream weakening effect of EA (NA) on the zonal flow that impinges on the other topography, which is mainly responsible for the much stronger interference of EA with NA's effects on the wave pattern than the other way around.

In general, the topographic forcing of EA can always modify the wave pattern across the Northern Hemisphere, regardless of the existence of the mountains of NA. The existence of NA helps to slightly extend the wavelength of the EA-forced flow pattern. In contrast, the topographic effects of NA on the wave pattern over Eurasia and the North Pacific can be greatly obstructed due to the existence of the EA. The much stronger interference of EA with NA's effect is related to EA's stronger downstream weakening effect on the zonal flow that impinges on NA. The joint effects of EA and NA on the tropospheric wave pattern is also generally dominated by the EA forcing.

\section{b. Upward wave propagations from the upper troposphere}

Wave pattern changes in the troposphere can result in changes in the upward wave propagation into the stratosphere. Shown in Fig. 7 are the vertical components of the three-dimensional wave activity flux and their changes due to topographic forcing. First, it is apparent that the four experiments consistently show that, in the northern extratropics in winter, three upward wave-flux action centers exist: the strongest upward center extending from EA to the eastern Pacific region, the second-strongest upward wave flux center over the North Atlantic-western Europe region, and the smaller downward wave-flux center over NA (Figs. 7a,b,d,e). Second, the independent topographic effect of EA is to force two upward wave-flux anomaly centers over the EA-western Pacific and North Atlantic regions, respectively (Fig. 7c), which lie correspondingly on the downstream side of the two anticyclonic high anomaly centers over Eurasia and NA, respectively, as shown in Fig. 5c. Meanwhile, downward wave-flux anomalies appear over the region from the Pacific to NA and over western Europe, which are respectively connected with the cyclonic low anomaly centers over the northwestern Pacific and over the Atlantic (Fig. 7c vs Fig. 5c). Clearly, the wave-flux anomalies forced by EA serve to increase the upward wave propagation over the EA and Atlantic regions significantly, but slightly decrease that over the Pacific and western Europe.

Although the two upward wave-flux anomaly centers downstream of the two anticyclonic anomaly centers over Eurasia and NA still exist when NA exists (Fig. 7f), a stronger downward wave-flux anomaly center appears from the eastern Pacific to the North American continent, which is related to the further-intensified and eastward-extended cyclonic anomaly centers (or a farther eastward-extended EAT and further-weakened NAR) (Figs. 5f and 7f). Comparing Fig. 7f with Fig. $7 \mathrm{c}$, we can see that the upward (downward) wave-flux anomalies are overall weakened (enhanced) due to the existence of NA, consistent with its destructive interference on the independent-EA-forced polar warming and polar vortex weakening.

It can be seen from Fig. $7 \mathrm{~g}$ that the independent effect of NA is mainly to enhance the upward wave flux over the eastern North Pacific, and that over the Eurasian continent, in relation to the deepened and eastwardextended EAT and the intensified NAR as indicated in Fig. 5g. Corresponding to the less intensification of the NAR and the less-changed EAT when EA exists (Fig. 5h), the enhancement of the upward wave flux over these regions becomes trivial, while the downward wave flux over NA begins to be slightly enhanced (Fig. 7h). This results in the overall downward wave-flux anomalies (shown in Fig. 8h) and thus the intensified stratospheric polar vortex and polar jet (Figs. $3 \mathrm{~h}$ and $4 \mathrm{~h}$ ). In addition, the wave-flux anomaly pattern forced by the joint effects of EA and NA (Fig. 7i) also resembles the independent-EA-forced effect (Fig. 7c), to a larger extent than the independentNA-forced effect (Fig. 7g). Comparing Fig. 7i with Fig. $7 \mathrm{c}$ and with Figs. $7 \mathrm{f}$ and $7 \mathrm{~g}$, it can be seen that the enhanced upward wave flux over the eastern North Pacific in Fig. 7i is mainly contributed by the independent-NA-forced effect, while the enhanced downward wave flux over the North American continent seems co-contributed by the NA-forced and EAforced effects when mutual interference is present (Fig. 7i vs Figs. 7f,h).

Overall, besides the obstructed effects of NA over Eurasia due to the existence of EA (Fig. $7 \mathrm{~g}$ vs Fig. $7 \mathrm{~h}$ ), the mutual interference between EA and NA is always to destruct the upward wave-flux anomalies over the eastern North Pacific region but to enhance the downward waveflux anomalies over the North American continent that are forced by either independent topography (Fig. 7c vs Fig. 7f; Fig. 7g vs Fig. 7h). This suggests the possible key regions of the mutual interference between EA and NA in their influences on the upward wave flux and on the stratosphere. Further investigations are obviously needed 
(a) noEA\&noNA



(d) noEA

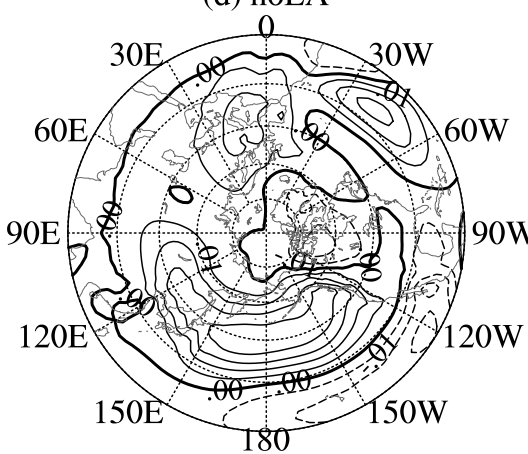

(g) (d)-(a)

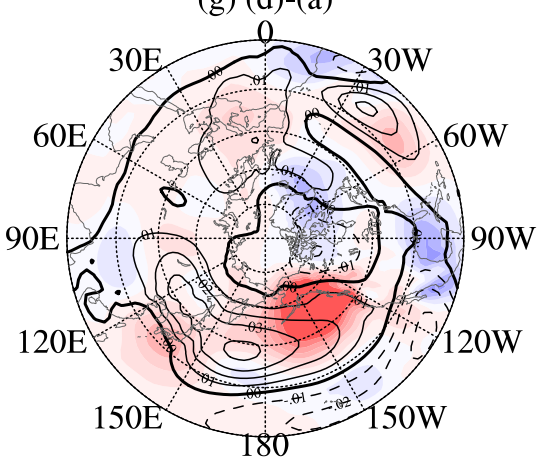

(b) noNA

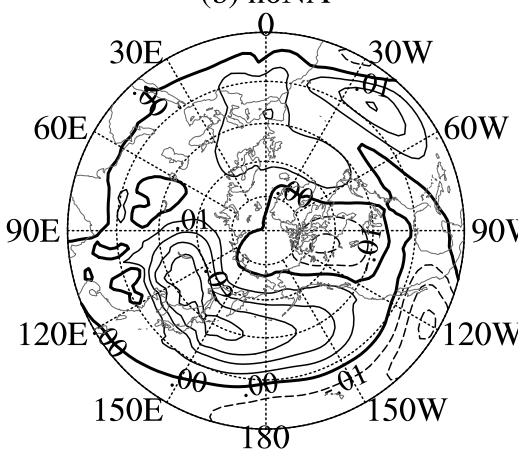

(e) CTL

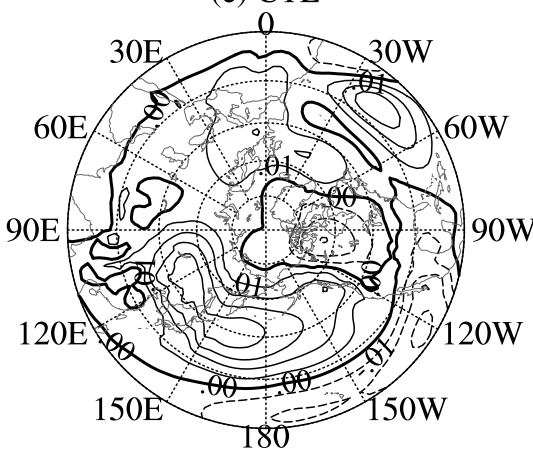

(h) (e)-(b)

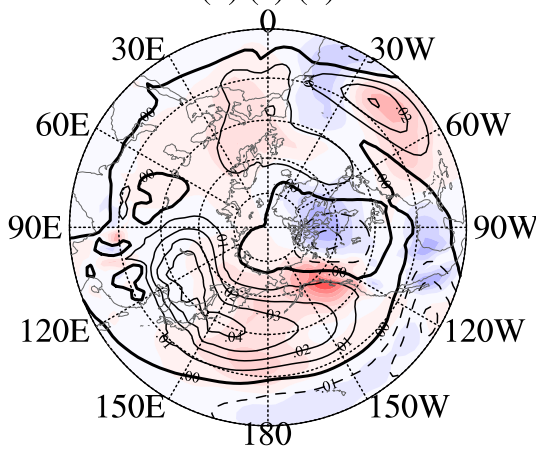

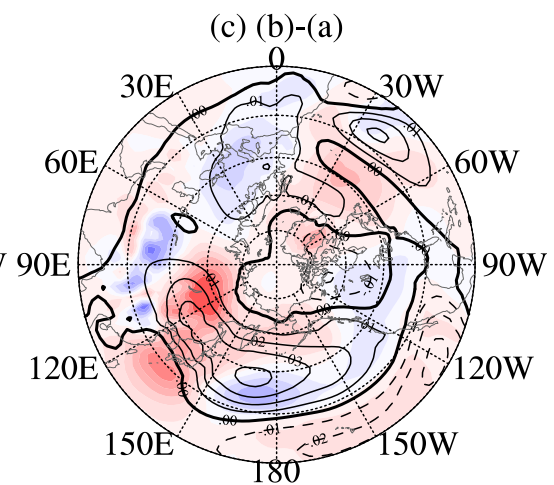

(f) (e)-(d)

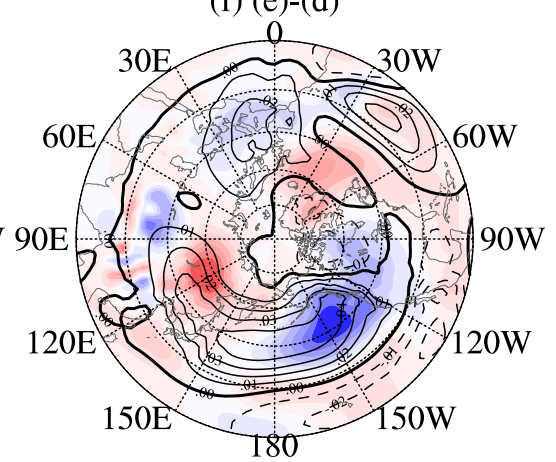

(i) (e)-(a)
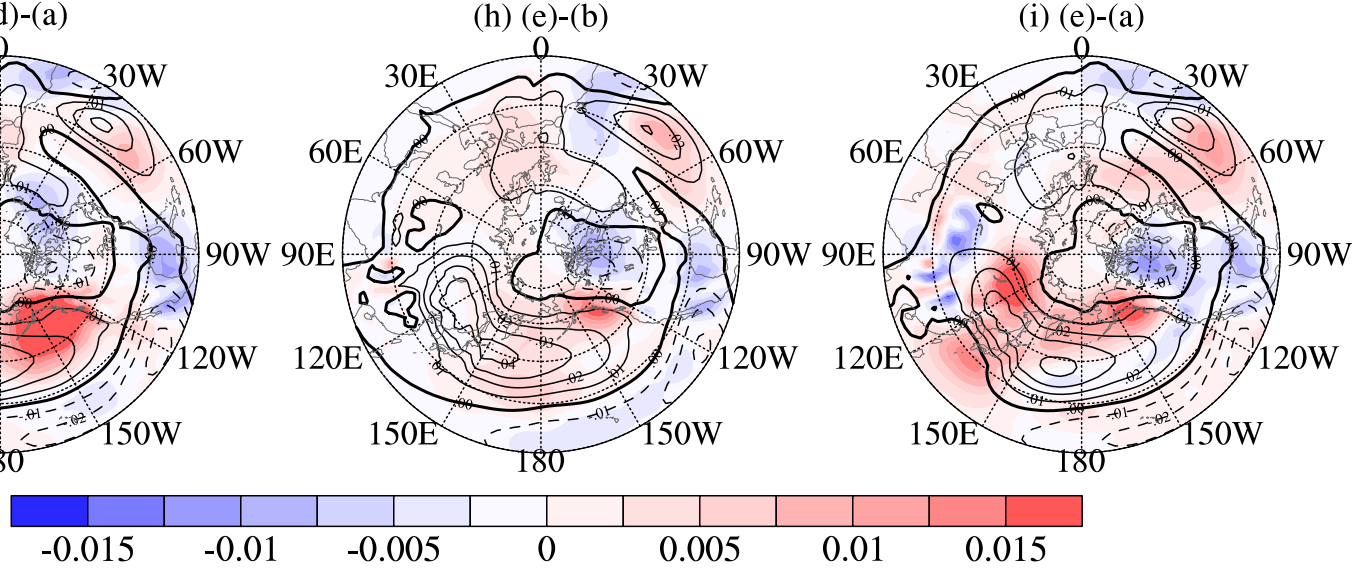

FIG. 7. As in Fig. 5, but for the vertical component of the three-dimensional wave activity fluxes $\left(\mathrm{m}^{2} \mathrm{~s}^{-2}\right.$; contours, interval: 0.01$)$. The contours in (c), (f), and (g)-(i) are also the winter climatology shown in (a), (d), (a), (b), and (a), respectively. Shading indicates the difference between different experiments.

for a full understanding of these key regions for the mutual interference within the topographic effects on the wave propagation.

\section{c. Zonal-mean EP flux patterns}

On top of the wave-flux pattern and its changes, the zonal-mean EP flux patterns and their differences between different experiments are shown in Fig. 8 . First, the four experiments consistently reproduce the zonal-mean upward EP flux in the extratropics from the troposphere to the stratosphere, and the EP flux convergence in the extratropical stratosphere (Figs. 8a,b,d,e). Second, consistent with the overall increase in upward wave flux shown in Fig. 7c, the independent effect of EA is indeed to induce the anomalously increased upward EP fluxes in the extratropics, which yields significant EP-flux convergence in the extratropical stratosphere (Fig. 8c). The regional decrease in upward wave flux over the eastern North Pacific and the increase in downward wave flux over the North American continent when NA exists (shown in Fig. 7f), does indeed correspond to the far lesser increase in upward EP flux in the extratropics than that without NA, despite weaker upward wave-flux anomalies still remaining in the 
(a) noEA\&noNA

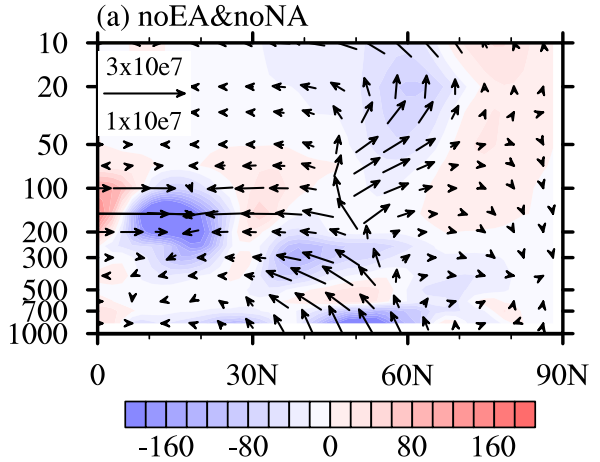

(d) noEA

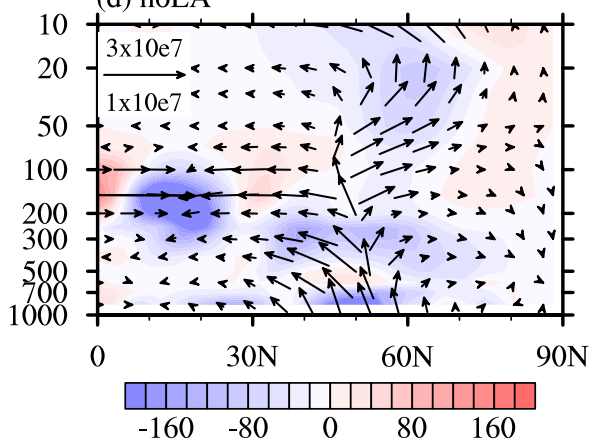

$\begin{array}{llllll}-160 & -80 & 0 & 80 & 160\end{array}$

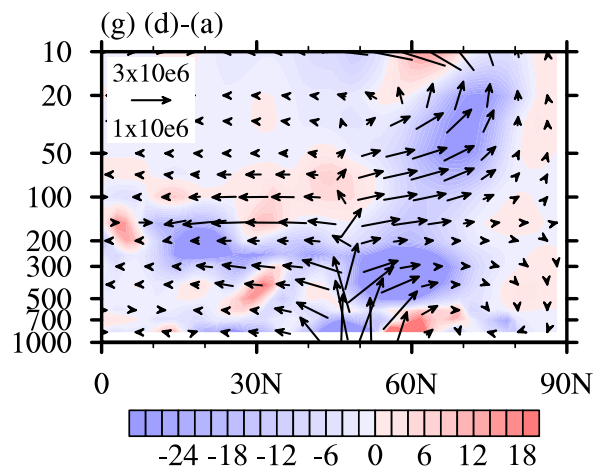

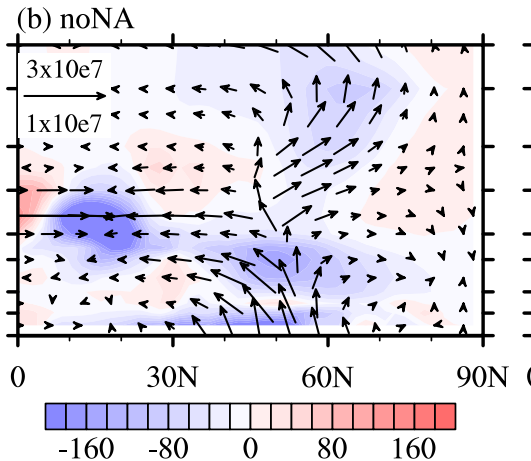

(e) CTL

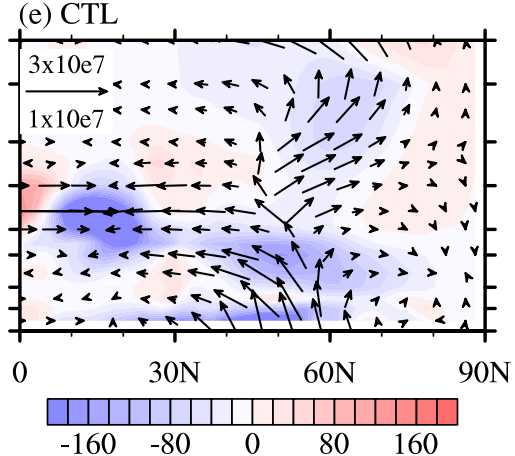



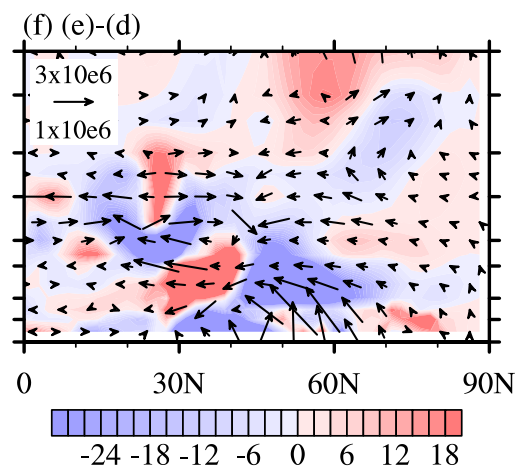



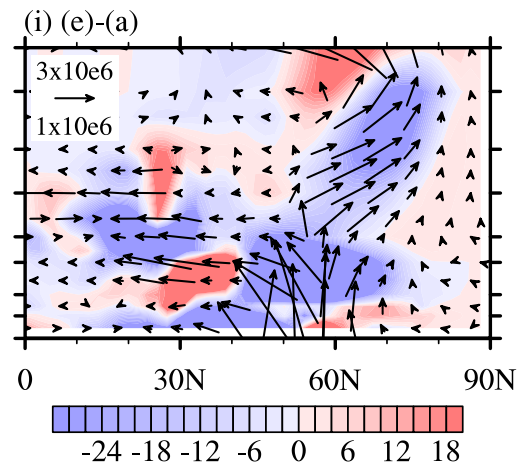

FIG. 8. As in Fig. 3, but for the zonal-mean EP flux $\left[\mathrm{kg}\left(\mathrm{m} \mathrm{s}^{-2}\right)^{-1}\right.$; vectors; amplified fourfold for those from $150 \mathrm{hPa}$ and above $]$ and its divergence $\left(\mathrm{m}^{2} \mathrm{~s}^{-2}\right.$; shading). The color bars for (a), (b), (d), and (e) are different from those for other panels.

midstratosphere (Fig. 8f vs Fig. 8c). Third, the much stronger regional increase in upward EP flux over the eastern North Pacific, as well as the globally extended independent effects of NA shown in Fig. 7g, corresponds to a significant increase in upward EP flux and its convergence in the extratropical stratosphere (Fig. 8g). When EA is not flattened, its obstructive effects on the independent-NA-forced wave pattern are seen from the weakest upward EP flux anomalies in the extratropical stratosphere, as well as a strong EP-flux divergence in the upper stratosphere (Fig. 8h). Finally, it can be seen from Fig. 8i that the upward EP flux and its convergence anomalies in the stratosphere, forced by the joint effects of EA and NA, are the strongest, which also exhibit a very similar pattern to that of the independent-EA-forced effect (Fig. 8i vs Fig. 8c), but are not a linear superimposition of their independent effects, as for those in previous figures. Moreover, it should be noted that the topographic effects always act to shift the main zone of upward EP flux and its convergence slightly poleward relative to its climatology (Figs. 8a,b,d,e vs Figs, 8c,f-i), which we attempt to investigate in the next section.

\section{d. Rossby stationary wavenumber}

Further examination of the EP-flux changes shown in Figs. $8 \mathrm{f}$ and $8 \mathrm{~h}$ clearly indicates that the decreased upward wave-flux anomalies relative to Figs. $8 \mathrm{c}$ and $8 \mathrm{~g}$ are always accompanied by increased equatorward wave fluxes in the extratropics, especially in the upper troposphere. To understand this, we show in Fig. 9 the 
(a) noEA\&noNA

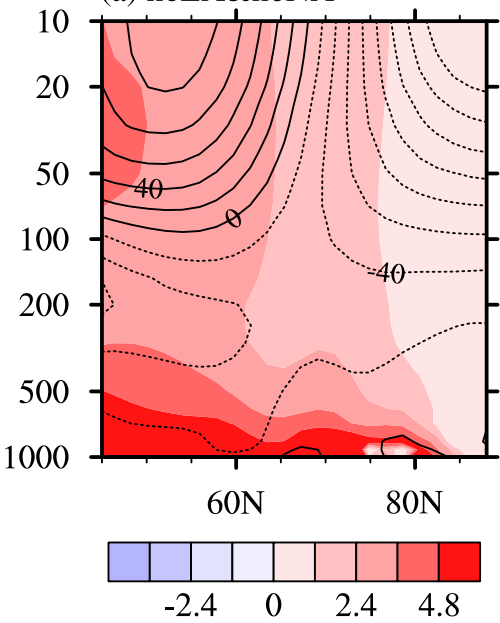

(d) noEA

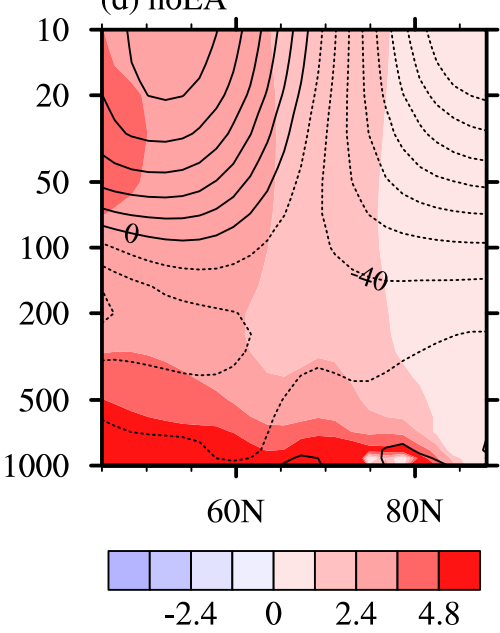

(g) (d)-(a)

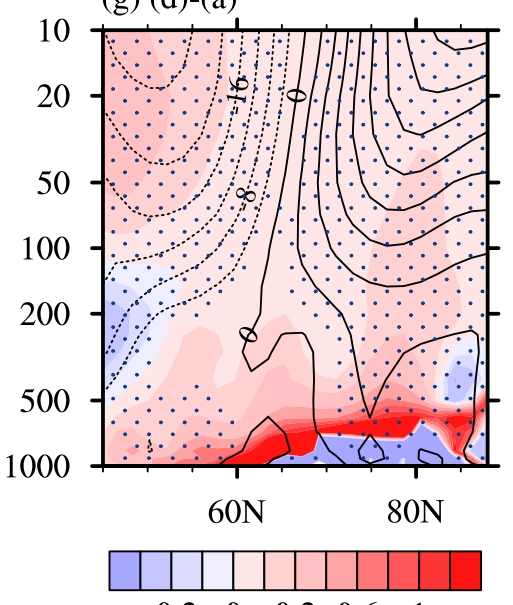

$\begin{array}{lllll}-0.2 & 0 & 0.2 & 0.6 & 1\end{array}$ (b) noNA

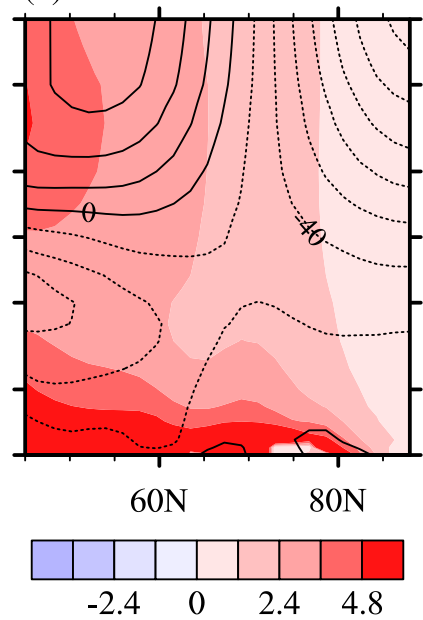

(e) CTL

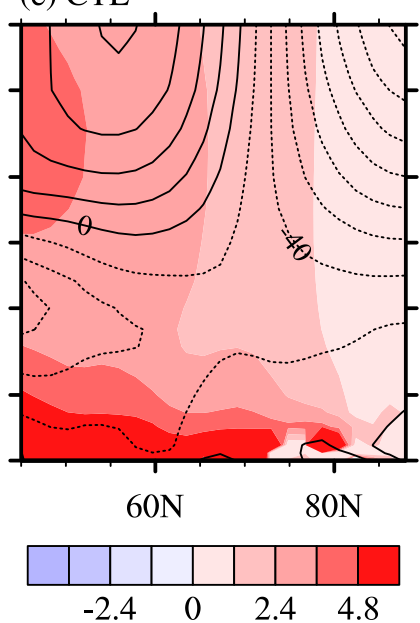

(h) (e)-(b)

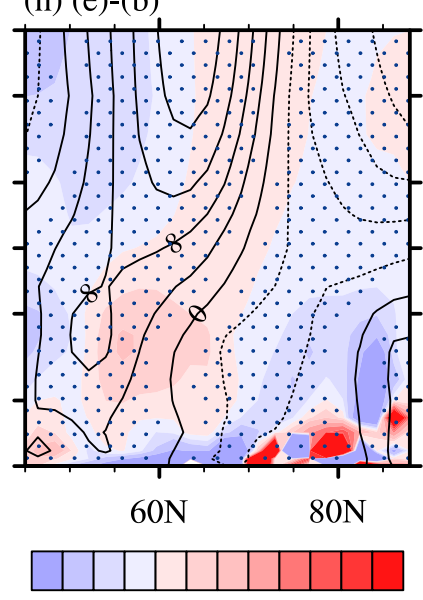

$\begin{array}{lllll}-0.2 & 0 & 0.2 & 0.6 & 1\end{array}$ (c) (b)-(a)

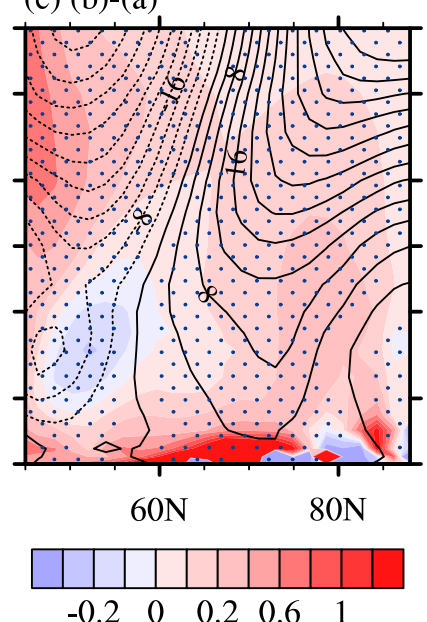

(f) (e)-(d)

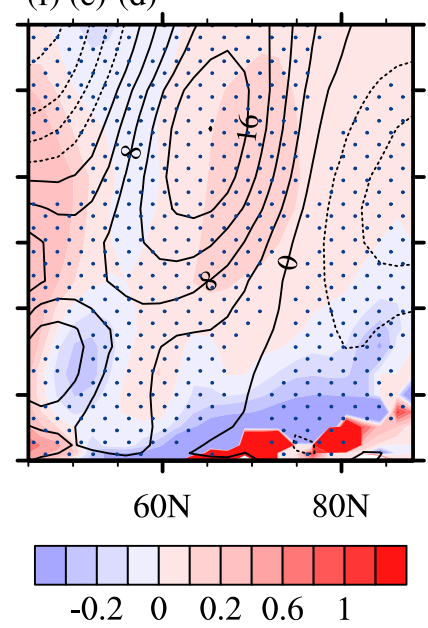

(i) (e)-(a)

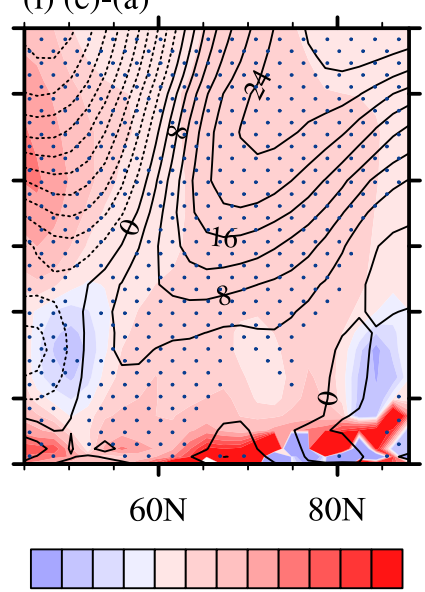

$\begin{array}{lllll}-0.2 & 0 & 0.2 & 0.6 & 1\end{array}$

FIG. 9. As in Fig. 8, but for the Rossby stationary wavenumber $k_{s}$ (shading) and the meridional gradient of zonalmean zonal wind (multiplied by $a$, Earth's radius; $\mathrm{m} \mathrm{s}^{-1}$; contours). The contour interval in (a), (b), (d), and (e) is 20 and that in (c), (f), and (g)-(i) is 4 . Dotted areas indicate the $95 \%$ confidence level of the difference in the meridional gradient of zonal-mean zonal wind from the Student's $t$ test. The color bars for (a), (b), (d), and (e) are different from those for other panels. 
Rossby stationary wavenumber $k_{s}$ and its difference between different experiments north of $40^{\circ} \mathrm{N}$. Also shown in Fig. 9 is the meridional gradient of the zonal-mean zonal wind ( $a \bar{U}_{y}$, where $a$ is the radius of Earth) and its corresponding changes due to topographic forcing. Based on the definition of $k_{s}$ in Eq. (4) as well as discussions in early studies (Hoskins and Ambrizzi 1993; Hoskins and Karoly 1981; Zou et al. 1991a,b), $k_{s}$ is closely related to $\bar{U}_{y}$, and should be proportional to $-d \bar{U}_{y} / d y$. In other words, large $k_{s}$ appears where $d \bar{U}_{y} / d y$ is at its negative peak (or where the maximum decrease in $\bar{U}_{y}$ with latitude occurs).

Related to the topography-forced weakening of the $\bar{U}$ from the troposphere to stratosphere (Figs. 3c,g,i), positive (negative) changes in $\bar{U}_{y}$ appear in the high latitudes (midlatitudes), which correspond to the positive (negative; or at least much more weakly positive) changes in $k_{s}$ on the poleward (equatorward) side of the positive $\bar{U}_{y}$ center, where the meridional gradient of $\bar{U}_{y}$ is relatively larger (Figs. 9c,g,i). The increased $k_{s}$ in the polar region thus explains the increase in poleward and upward wave propagation, and therefore the weakening of the stratospheric polar vortex forced by the independent EA and NA, as well as their joint forcing shown in previous figures (Figs. 3, 7, and 8). When the mutual interference between EA and NA is considered, negative changes in $k_{s}$ appear in the high latitudes, especially in the troposphere (Figs. 9f,h), which can be attributed to the negative changes in $\bar{U}_{y}$ in the polar region, which in turn is associated with the westerly anomalies in the subpolar region from the troposphere to the lower stratosphere (Figs. 3f,h). The decrease in stationary wavenumber in high latitudes thus favors an increase in equatorward wave propagation, which helps to reduce the upward propagation of planetary waves in the high latitudes (as seen from the EP flux anomalies in Figs. 8f and 8h). In general, the overall changes in the upward wave propagation into the extratropical stratosphere between different experiments are largely determined by the topography-forced changes in the zonal-mean pattern of the zonal flow in the extratropics, which implies a different meridional distribution of the Rossby stationary wavenumber $k_{s}$. Nevertheless, because inevitably the changes in upward wave propagation will in turn influence the zonal flow pattern, the cause and effect between them is still hard to separate in the current regime.

Furthermore, since the positive changes in $k_{s}$ always lie in the polar region north of $60^{\circ} \mathrm{N}$ (Figs. $9 \mathrm{c}, \mathrm{f}-\mathrm{i}$ ), the topography-forced anomalous upward wave propagation, or the anomalous EP flux and its convergence, thus always tend to appear north of their climatological mean zone (around $60^{\circ} \mathrm{N}$ ), and favor a poleward shift of the mean zone (Fig. 8). In relation to the much higher latitudes that NA extends to, the NA-forced maximum changes in $\bar{U}_{y}$ (and $k_{s}$ ) in the lower troposphere appear at a relatively higher latitude than the EA-forced ones (e.g., Fig. 9c vs Fig. 9g). On the other hand, the NAforced changes in $\bar{U}_{y}$ (and $k_{s}$ ) are relatively weaker than the EA-forced ones in the polar stratosphere (e.g., Fig. 9c vs Fig. 9g) due to the much weaker topographic forcing of NA, which implies much weaker upward wave propagation or weaker poleward eddy heat transport. Weaker poleward eddy heat transport thus corresponds to a lesser poleward progression of the maximum changes in $\bar{U}_{y}$ with altitude or a lesser vertical tilting of the $\bar{U}_{y}$ axis. Therefore, the NA-forced (EA-forced) zonal wind pattern exhibits a relatively more "barotropic" ("baroclinic") structure, as shown in Fig. 3.

\section{Summary and discussion}

By performing a series of numerical experiments with the stratosphere-resolving WACCM, this study first demonstrates the independent topographic effects of EA and NA, and then compares them with their dependent effects to demonstrate the mutual interference between them within their topographic effects in the extratropical stratosphere. The results show that, contrary to the known much weaker topographic effect of NA than EA on the planetary wave activity and on the stratospheric polar vortex, the intensity of NA's effect turns out to be comparable with EA's in modulating the circulation pattern from the troposphere to the stratosphere when the NA's topography is as an independent forcing without EA. It can also, like EA, induce severe polar warming and weakening of the stratospheric polar vortex. While EA favors a displacement of the stratospheric polar vortex toward Eurasia, NA favors a displacement toward the NA-Atlantic region. However, when the topographic forcing of either EA or NA is set to be not independent but with the other present, significant mutual destructive interference is found. Specifically, the independent-EA-forced weakening of the stratospheric polar vortex is highly decreased and changes to a main location displacement of the polar vortex when NA exists. The interference the other way around is even more critical, tending to completely offset the independent-NA-forced effect to a strengthening of the stratospheric polar vortex and polar jet. The much weaker and less significant impacts of NA on the stratosphere indicated in previous studies can be explained by the strong interference of EA.

EA can always modulate the global wave pattern significantly, including that over NA, regardless of the existence of NA's topography. The existence of NA helps to extend the wavelength of the EA-forced flow 
TABLE 2. Summary of the main facts of the topographic effects in the extratropical stratosphere.

\begin{tabular}{|c|c|c|c|}
\hline Topography & Independent forcing & Dependent forcing & Mutual interference \\
\hline EA & $\begin{array}{l}\text { (i) Severe stratospheric polar } \\
\text { warming and polar vortex } \\
\text { weakening. } \\
\text { (ii) Polar vortex is slightly displaced } \\
\text { toward Eurasia. }\end{array}$ & $\begin{array}{l}\text { (i) Highly weakened polar } \\
\text { warming and polar vortex } \\
\text { weakening. } \\
\text { (ii) Polar vortex is mainly displaced } \\
\text { toward Eurasia. }\end{array}$ & $\begin{array}{l}\text { (i) May completely offset the } \\
\text { independent-NA-forced effect in the } \\
\text { stratosphere. } \\
\text { (ii) Can obstruct NA's effect on the } \\
\text { tropospheric wave pattern over the } \\
\text { Eurasia-Pacific region due to a much } \\
\text { stronger downstream weakening effect } \\
\text { on the zonal flow impinging on NA. }\end{array}$ \\
\hline NA & $\begin{array}{l}\text { (i) Severe stratospheric polar } \\
\text { warming and polar vortex } \\
\text { weakening comparable with the } \\
\text { EA-forced effect. } \\
\text { (ii) Polar vortex displacement } \\
\text { toward the NA-Atlantic region. }\end{array}$ & $\begin{array}{l}\text { (ii) Influences on the upper- } \\
\text { tropospheric flow pattern are } \\
\text { confined within the NA- } \\
\text { Atlantic region. }\end{array}$ & $\begin{array}{l}\text { (i) Can highly weaken the independent- } \\
\text { EA-forced polar warming and increase } \\
\text { the polar vortex displacement toward } \\
\text { Eurasia. } \\
\text { (ii) Can extend wavelength of the } \\
\text { independent-EA-forced flow pattern } \\
\text { in the upper troposphere. }\end{array}$ \\
\hline EA\&NA & \multicolumn{2}{|c|}{$\begin{array}{l}\text { (i) Severe stratospheric polar warming and polar vortex weakening, } \\
\text { and not a linear superimposition but dominated by the } \\
\text { independent-EA-forced effect. } \\
\text { (ii) Highly similar flow pattern to the independent-EA-forced one. }\end{array}$} & $\begin{array}{l}\text { (i) Destruction of the upward wave flux } \\
\text { over the eastern North Pacific and/or } \\
\text { (ii) enhancement of the downward wave } \\
\text { flux over the North American } \\
\text { continent. }\end{array}$ \\
\hline
\end{tabular}

pattern. In contrast, the effect of NA on the circulation pattern over the EA-Pacific region can be substantively obstructed when EA exists. In other words, NA's effect is largely confined to a limited downstream region over the NA-Atlantic region due to the existence of EA. The much stronger interference of EA with the effects of NA compared with the other way around is related to the much stronger downstream weakening effect of EA on the zonal flow that impinges on NA. While the independent topographic effects of EA and NA both act to increase the upward wave flux into the stratosphere, mutual interference always tends to further suppress the upward wave flux, mainly over the eastern North Pacific region, and increase the downward wave flux, mainly over the North American continent, thus resulting in an overall decrease in the upward propagation of planetary waves into the stratosphere. On the other hand, the overall changes in planetary wave propagation can also be explained by the topography-forced changes in the zonalmean pattern of zonal flow, which yield the changes in the meridional distribution of the Rossby stationary wavenumber $k_{s}$. Since the topography-forced increase in $k_{s}$ always lies in the polar region north of $60^{\circ} \mathrm{N}$, the anomalous upward EP flux and its divergence always tend to appear north of the climatological mean zone (around $60^{\circ} \mathrm{N}$ ) and shift it poleward, when topographic forcing is present.

In relation to the much stronger interference of EA with NA's effect, the joint effect when EA and NA are incorporated simultaneously is not a linear superimposition of their independent effects but largely dominated by EA's effect wherein a severe weakening of the stratospheric polar vortex and polar jet is induced. To provide a general picture of the topographic effects from EA and NA, we summarize in Table 2 the main facts of the independent, dependent, and joint effects from EA and NA, as well as the mutual interference between them.

In short, this study has provided some new evidence of the mutual interference between EA and NA in their influences on the extratropical stratosphere and the related flow patterns in the troposphere. The importance of distinction between the independent, dependent, and joint topographic effects is emphasized, for a possible full understanding of the various topographic effects from different regions, in the extratropical stratosphere and troposphere. The findings of this study may help to advance our knowledge of the main topographic forcing that helps to formulate the leading circulation and climate patterns in winter and their changes. Obviously, the approach of this study can be adopted for studying the mutual interference between different regions, of the various topographic effects including that in the lower troposphere, and potentially help to advance our understanding of the various climate effects of large-scale mountains (including EA and NA) and other plateaus and mountains as already indicated in White et al. (2018). The future change in these topographic effects when the zonal westerly jet is meridionally shifted is another important aspect that should be investigated.

Furthermore, we currently consider EA's topography as a whole, making no distinction between the Tibetan 
Plateau, Iranian Plateau, and Mongolian Plateau. This aspect, in terms of the possible interference between each of these plateaus and the mountains of NA, should be investigated in the future. Comparing the results in White et al. (2018) (see their Fig. 2) with the results in this study (see Fig. 3 herein), we can see that there must also exist a mutual interaction between the Tibetan Plateau and the Mongolia Plateau, which requires further investigation. In addition, our preliminary statistics indicate that the occurrence of sudden stratospheric warming (SSW) events might be significantly influenced by different topographic forcing. For example, both an independent EA and independent NA may increase the occurrence of SSWs of mainly the vortex-displacement type; the mutual interference between them always tends to increase the vortex-split type but decrease the vortexdisplacement type of SSWs; and the joint forcing of EA\&NA may increase both types (not shown). Therefore, the above topographic effects on stratospheric variabilities, including the dominant intraseasonal time scale oscillation, stratosphere-troposphere coupling, and downward stratospheric influences in the extratropics, should also be investigated in the future.

Acknowledgments. All authors thank the editor and the three anonymous reviewers for their insightful and constructive suggestions and comments, which helped us to substantially improve and strengthen the paper. This work was jointly supported by the Strategic Priority Research Program of Chinese Academy of Sciences (XDA17010105), the National Science Foundation of China (projects 91837311, 41575041, 91437105, 41705024 and 41430533), and the Key Research Program of Frontier Sciences (QYZDY-SSWDQC018). We are grateful for the availability of WACCM, provided by NCAR via the website http:// www.cesm.ucar.edu/models/current.html.

\section{REFERENCES}

Andrews, D., and M. E. McIntyre, 1976: Planetary waves in horizontal and vertical shear: The generalized Eliassen-Palm relation and the mean zonal acceleration. J. Atmos. Sci., 33, 2031-2048, https://doi.org/10.1175/1520-0469(1976)033<2031: PWIHAV $>2.0 . \mathrm{CO} ; 2$.

Baldwin, M. P., and T. J. Dunkerton, 1999: Propagation of the Arctic Oscillation from the stratosphere to the troposphere. J. Geophys. Res., 104, 30 937-30 946, https://doi.org/10.1029/ 1999JD900445.

—, and —, 2001: Stratospheric harbingers of anomalous weather regimes. Science, 294, 581-584, https://doi.org/ 10.1126/science.1063315.

Cai, M., 2003: Potential vorticity intrusion index and climate variability of surface temperature. Geophys. Res. Lett., 30, 1119, https://doi.org/10.1029/2002GL015926.
— , Y. Yu, Y. Deng, H. M. van den Dool, R. Ren, S. Saha, X. Wu, and J. Huang, 2016: Feeling the pulse of the stratosphere: An emerging opportunity for predicting continental-scale cold-air outbreaks 1 month in advance. Bull. Amer. Meteor. Soc., 97, 1475-1489, https://doi.org/10.1175/BAMS-D-14-00287.1.

Calvo, N., R. Garcia, W. Randel, and D. Marsh, 2010: Dynamical mechanism for the increase in tropical upwelling in the lowermost tropical stratosphere during warm ENSO events. J. Atmos. Sci., 67, 2331-2340, https://doi.org/10.1175/2010JAS3433.1.

Chang, E. K. M., 2009: Diabatic and orographic forcing of northern winter stationary waves and storm tracks. J. Climate, 22, 670688, https://doi.org/10.1175/2008JCLI2403.1.

Charlton-Perez, A. J., and Coauthors, 2013: On the lack of stratospheric dynamical variability in low-top versions of the CMIP5 models. J. Geophys. Res. Atmos., 118, 2494-2505, https://doi.org/10.1002/jgrd.50125.

Chen, S.-C., and K. E. Trenberth, 1988: Forced planetary waves in the Northern Hemisphere winter: Wave-coupled orographic and thermal forcings. J. Atmos. Sci., 45, 682-704, https://doi.org/ 10.1175/1520-0469(1988)045<0682:FPWITN > 2.0.CO;2.

Chen, W., X. Lan, L. Wang, and Y. Ma, 2013: The combined effects of the ENSO and the Arctic Oscillation on the winter climate anomalies in East Asia. Chin. Sci. Bull., 58, 1355-1362, https:// doi.org/10.1007/s11434-012-5654-5.

Cook, K. H., and I. M. Held, 1992: The stationary response to largescale orography in a general circulation model and a linear model. J. Atmos. Sci., 49, 525-539, https://doi.org/10.1175/ 1520-0469(1992)049<0525:TSRTLS > 2.0.CO;2.

de la Torre, L., R. R. Garcia, D. Barriopedro, and A. Chandran, 2012: Climatology and characteristics of stratospheric sudden warmings in the Whole Atmosphere Community Climate Model. J. Geophys. Res., 117, D04110, https://doi.org/10.1029/ 2011JD016840.

Ding, Y., 1992: Effects of the Qinghai-Xizang (Tibetan) plateau on the circulation features over the plateau and its surrounding areas. Adv. Atmos. Sci., 9, 112-130, https://doi.org/10.1007/BF02656935.

Eyring, V., and Coauthors, 2007: Multimodel projections of stratospheric ozone in the 21st century. J. Geophys. Res., 112 D16303, https://doi.org/10.1029/2006JD008332.

_ , and Coauthors, 2010: Multi-model assessment of stratospheric ozone return dates and ozone recovery in CCMVal-2 models. Atmos. Chem. Phys., 10, 9451-9472, https://doi.org/ 10.5194/acp-10-9451-2010.

Garcia, R., D. Marsh, D. Kinnison, B. Boville, and F. Sassi, 2007: Simulation of secular trends in the middle atmosphere, 19502003. J. Geophys. Res., 112, D09301, https://doi.org/10.1029/ 2006JD007485.

Garfinkel, C. I., D. L. Hartmann, and F. Sassi, 2010: Tropospheric precursors of anomalous Northern Hemisphere stratospheric polar vortices. J. Climate, 23, 3282-3299, https://doi.org/ 10.1175/2010JCLI3010.1.

Gerber, E. P., and L. M. Polvani, 2009: Stratosphere-troposphere coupling in a relatively simple AGCM: The importance of stratospheric variability. J. Climate, 22, 1920-1933, https:// doi.org/10.1175/2008JCLI2548.1.

Held, I. M., 1983: Stationary and quasi-stationary eddies in the extratropical troposphere: Theory. Large-Scale Dynamical Processes in the Atmosphere, B. Hoskins and R. P. Pearce, Eds., Academic Press, 127-168.

— , and M. Ting, 1990: Orographic versus thermal forcing of stationary waves: The importance of the mean low-level wind. J. Atmos. Sci., 47, 495-500, https://doi.org/10.1175/15200469(1990)047<0495:OVTFOS $>2.0$.CO;2. 
__, _ - and H. Wang, 2002: Northern winter stationary waves: Theory and modeling. J. Climate, 15, 21252144, https://doi.org/10.1175/1520-0442(2002)015<2125: NWSWTA $>2.0 . \mathrm{CO} ; 2$.

Hoskins, B. J., and D. J. Karoly, 1981: The steady linear response of a spherical atmosphere to thermal and orographic forcing. J. Atmos. Sci., 38, 1179-1196, https://doi.org/10.1175/15200469(1981)038<1179:TSLROA > 2.0.CO;2.

_ , and T. Ambrizzi, 1993: Rossby wave propagation on a realistic longitudinally varying flow. J. Atmos. Sci., 50, 1661-1671, https://doi.org/10.1175/1520-0469(1993)050<1661: RWPOAR $>2.0 . \mathrm{CO} ; 2$.

Jackman, C. H., D. R. Marsh, F. M. Vitt, R. R. Garcia, C. E. Randall, E. L. Fleming, and S. M. Frith, 2009: Long-term middle atmospheric influence of very large solar proton events. J. Geophys. Res., 114, D11304, https://doi.org/10.1029/ 2008JD011415.

Jacqmin, D., and R. Lindzen, 1985: The causation and sensitivity of the northern winter planetary waves. J. Atmos. Sci., 42, 724-746, https:// doi.org/10.1175/1520-0469(1985)042<0724:TCASOT>2.0.CO;2.

Kasahara, A., and W. M. Washington, 1971: General circulation experiments with a six-layer NCAR model, including orography, cloudiness and surface temperature calculations. J. Atmos. Sci., 28, 657-701, https://doi.org/10.1175/ 1520-0469(1971)028<0657:GCEWAS > 2.0.CO;2.

Limpasuvan, V., J. H. Richter, Y. J. Orsolini, F. Stordal, and O.-K. Kvissel, 2012: The roles of planetary and gravity waves during a major stratospheric sudden warming as characterized in WACCM. J. Atmos. Sol-Terr. Phys., 78-79, 84-98, https:// doi.org/10.1016/j.jastp.2011.03.004.

Lin, B.-D., 1982: The behavior of winter stationary planetary waves forced by orography and diabatic heating. J. Atmos. Sci., 39, 1206-1226, https://doi.org/10.1175/1520-0469(1982)039<1206: TBOWSP $>2.0 . \mathrm{CO} ; 2$.

Lott, F., L. Fairhead, F. Hourdin, and P. Levan, 2005: The stratospheric version of LMDz: Dynamical climatologies, Arctic Oscillation, and impact on the surface climate. Climate Dyn., 25, 851-868, https://doi.org/10.1007/ s00382-005-0064-x.

Luo, M., B. Zhu, and X. Zhang, 1985: The dynamic effect of the Tibetan Plateau on the formation of zonal type circulation over East Asia. Adv. Atmos. Sci., 2, 158-166, https://doi.org/ 10.1007/BF03179748.

Manabe, S., and T. B. Terpstra, 1974: The effects of mountains on the general circulation of the atmosphere as identified by numerical experiments. J. Atmos. Sci., 31, 3-42, https://doi.org/ 10.1175/1520-0469(1974)031<0003:TEOMOT >2.0.CO;2.

Marsh, D. R., R. R. Garcia, D. E. Kinnison, B. A. Boville, F. Sassi, S. C. Solomon, and K. Matthes, 2007: Modeling the whole atmosphere response to solar cycle changes in radiative and geomagnetic forcing. J. Geophys. Res., 112, D23306, https:// doi.org/10.1029/2006JD008306.

_- M. J. Mills, D. E. Kinnison, J.-F. Lamarque, N. Calvo, and L. M. Polvani, 2013: Climate change from 1850 to 2005 simulated in CESM1(WACCM). J. Climate, 26, 7372-7391, https://doi.org/10.1175/JCLI-D-12-00558.1.

Matthes, K., D. R. Marsh, R. R. Garcia, D. E. Kinnison, F. Sassi, and S. Walters, 2010: Role of the QBO in modulating the influence of the 11 year solar cycle on the atmosphere using constant forcings. J. Geophys. Res., 115, D18110, https:// doi.org/10.1029/2009JD013020.

Nakamura, H., 1978: Dynamical effects of mountains on the general circulation of the atmosphere: II. Effects of mountain barrier on a barotropic jet. J. Meteor. Soc. Japan, 56, 341-352, https://doi.org/10.2151/JMSJ1965.56.5_341.

Nigam, S., I. M. Held, and S. W. Lyons, 1986: Linear simulation of the stationary eddies in a general circulation model. Part I: The nomountain model. J. Atmos. Sci., 43, 2944-2961, https://doi.org/ 10.1175/1520-0469(1986)043<2944:LSOTSE > 2.0.CO;2.

$\ldots, \ldots$, and 1988: Linear simulation of the stationary eddies in a GCM. Part II: The "mountain" model. J. Atmos. Sci., 45, 1433-1452, https://doi.org/10.1175/1520-0469(1988) 045<1433:LSOTSE $>2.0$. CO;2.

Plumb, R. A., 1985: On the three-dimensional propagation of stationary waves. J. Atmos. Sci., 42, 217-229, https://doi.org/ 10.1175/1520-0469(1985)042<0217:OTTDPO>2.0.CO;2.

Rao, J., and R.-C. Ren, 2016a: A decomposition of ENSO's impacts on the northern winter stratosphere: Competing effect of SST forcing in the tropical Indian Ocean. Climate Dyn., 46, 3689-3707, https://doi.org/10.1007/s00382-015-2797-5.

, and - 2016b: Asymmetry and nonlinearity of the influence of ENSO on the northern winter stratosphere: 2. Model study with WACCM.J. Geophys. Res., 121, 9017-9032, https:// doi.org/10.1002/2015JD024521.

_ _ - and Y. Yang, 2015: Parallel comparison of the northern winter stratospheric circulation in reanalysis and in CMIP5 models. Adv. Atmos. Sci., 32, 952-966, https://doi.org/10.1007/ s00376-014-4192-2.

Rayner, N. A., D. E. Parker, E. B. Horton, C. K. Folland, L. V. Alexander, D. P. Rowell, E. C. Kent, and A. Kaplan, 2003: Global analyses of sea surface temperature, sea ice, and night marine air temperature since the late nineteenth century. J. Geophys. Res., 108, 4407, https://doi.org/10.1029/ 2002JD002670.

Ren, R.-C., J. Rao, G. X. Wu, and M. Cai, 2017: Tracking the delayed response of the northern winter stratosphere to ENSO using multi reanalyses and model simulations. Climate Dyn., 48, 2859-2879, https://doi.org/10.1007/s00382-016-3238-9.

Richter, J. H., F. Sassi, and R. R. Garcia, 2010: Toward a physically based gravity wave source parameterization in a general circulation model. J. Atmos. Sci., 67, 136-156, https://doi.org/ 10.1175/2009JAS3112.1.

Ringler, T., and K. Cook, 1995: Orographically induced stationary waves: Dependence on latitude. J. Atmos. Sci., 52, 2548-2560, https://doi.org/10.1175/1520-0469(1995)052<2548: OISWDO $>2.0 . \mathrm{CO} ; 2$.

Sassi, F., D. Kinnison, B. Boville, R. Garcia, and R. Roble, 2004: Effect of El Niño-Southern Oscillation on the dynamical, thermal, and chemical structure of the middle atmosphere. J. Geophys. Res., 109, D17108, https://doi.org/10.1029/ 2003JD004434.

Shi, Z. G., X. D. Liu, Y. M. Liu, Y. Y. Sha, and T. T. Xu, 2015: Impact of Mongolian Plateau versus Tibetan Plateau on the westerly jet over North Pacific Ocean. Climate Dyn., 44, 30673076, https://doi.org/10.1007/s00382-014-2217-2.

Smagorinsky, J., 1953: The dynamical influence of large-scale heat sources and sinks on the quasi-stationary mean motions of the atmosphere. Quart. J. Roy. Meteor. Soc., 79, 342-366, https:// doi.org/10.1002/qj.49707934103.

Smith, A. K., R. Garcia, D. R. Marsh, and J. H. Richter, 2011: WACCM simulations of the mean circulation and trace species transport in the winter mesosphere. J. Geophys. Res., 116, D20115, https://doi.org/10.1029/2011JD016083.

Taguchi, M., and S. Yoden, 2002: Internal interannual variability of the troposphere-stratosphere coupled system in a simple global circulation model. Part I: Parameter sweep experiment. 
J. Atmos. Sci., 59, 3021-3036, https://doi.org/10.1175/15200469(2002)059<3021:IIVOTT>2.0.CO;2.

and D. L. Hartmann, 2006: Increased occurrence of stratospheric sudden warmings during El Niño as simulated by WACCM. J. Climate, 19, 324-332, https://doi.org/10.1175/ JCLI3655.1.

Ting, M. F., H. Wang, and L. Yu, 2001: Nonlinear stationary wave maintenance and seasonal cycle in the GFDL R30 GCM. J. Atmos. Sci., 58, 2331-2354, https://doi.org/10.1175/15200469(2001)058<2331:NSWMAS > 2.0.CO;2.

Valdes, P. J., and B. J. Hoskins, 1991: Nonlinear orographically forced planetary waves. J. Atmos. Sci., 48, 2089-2106, https://doi.org/ 10.1175/1520-0469(1991)048<2089:NOFPW>2.0.CO;2.

Watanabe, S., Y. Kawatani, Y. Tomikawa, K. Miyazaki, M. Takahashi, and K. Sato, 2008: General aspects of a T213L256 middle atmosphere general circulation model. J. Geophys. Res., 113, D12110, https://doi.org/10.1029/2008JD010026.

White, R. H., D. S. Battisti, and G. H. Roe, 2017: Mongolian mountains matter most: Impacts of the latitude and height of Asian orography on Pacific wintertime atmospheric circulation. J. Climate, 30, 4065-4082, https://doi.org/10.1175/JCLI-D-16-0401.1.

, - - and A. Sheshadri, 2018: Orography and the boreal winter stratosphere: The importance of the Mongolian mountains. Geophys. Res. Lett., 45, 2088-2096, https://doi.org/ 10.1002/2018GL077098.

Xia, X., R. C. Ren, and Y. Y. Yu, 2019: Dynamical role of the Rocky Mountain controlled by East Asian topographies in modulating the tropospheric westerly jet in northern winter. Atmos. Oceanic Sci., 12, 66-72, https://doi.org/10.1080/ 16742834.2018.1548247.

Yanai, M., and G.-X. Wu, 2006, Effects of the Tibetan Plateau. The Asian Monsoon, Springer, 513-549, https://doi.org/10.1007/3540-37722-0_13.

Yu, Y., R. Ren, J. Hu, and G. Wu, 2014: A mass budget analysis on the interannual variability of the polar surface pressure in the winter season. J. Atmos. Sci., 71, 3539-3553, https://doi.org/ 10.1175/JAS-D-13-0365.1.

Zou, X., D. Ye, and G. Wu, 1991a: The analysis of dynamic effects on winter circulation of the two main mountains in the Northern Hemisphere I. Circulation, teleconnection and horizontal propagation of stationary waves (in Chinese). Acta Meteor. Sin., 49, 129-140.

G. Wu, and D. Ye, 1991b: The analysis of dynamic effects on winter circulation of the two main mountains in the Northern Hemisphere II. Vertical propagation of planetary waves (in Chinese). Acta Meteor. Sin., 49, 257-268. 